\title{
Supramolecular Plastics Processed from Small Molecule-based
}

\section{Coacervates}

Jingjing Yu ${ }^{1}$, Dawei Qi ${ }^{1}$, Ermei Mäkilä ${ }^{2}$, Lippo Lassila ${ }^{3}$, Anastassios C. Papageorgiou ${ }^{4,5}$, Markus Peurla $^{6}$, Jessica M. Rosenholm ${ }^{7}$, Zhao Zhao ${ }^{1}$, Pekka Vallittu ${ }^{3,8}$, Sirpa Jalkanen ${ }^{1}$, Chunman Jia ${ }^{9,10,}$ Jianwei $\mathrm{Li}^{1,9,10 *}$

${ }^{I}$ MediCity Research Laboratory, University of Turku, Tykistökatu 6, 20520 Turku, Finland

${ }^{2}$ Laboratory of Industrial Physics, Department of Physics and Astronomy, University of Turku, 20014 Turku, Finland

${ }^{3}$ Department of Biomaterials Science and Turku Clinical Biomaterials Centre - TCBC, Institute of Dentistry, University of Turku, 20014 Turku, Finland

${ }^{4}$ Turku Bioscience Centre, University of Turku, 20521 Turku, Finland

${ }^{5}$ Turku Bioscience Centre, Åbo Akademi University, 20521 Turku, Finland

${ }^{6}$ Institute of Biomedicine and FICAN West Cancer Research Laboratories, University of Turku, 20014 Turku, Finland

${ }^{7}$ Pharmaceutical Sciences Laboratory, Faculty of Science and Engineering, Åbo Akademi University, Tykistökatu 6, 20520 Turku, Finland

${ }^{8}$ City of Turku Welfare Division, Puolalankatu 5, 20101 Turku, Finland

${ }^{9}$ Hainan Provincial Key Laboratory of Fine Chem, School of Chemical Engineering and Technology, Hainan University, 570228 Haikou, China.

${ }^{10}$ One Health Institute, Hainan University, 570228 Haikou, China.

* Corresponding author, e-mail: Jianwei.li@utu.fi

\begin{abstract}
Supramolecular polymers self-assembled by small molecules are a type of new materials with adaptive and biocompatible properties. However, the mechanical property of these materials is always weak, impeding their applications in practice. Here, we reported that coacervation can be used to explore robust and stable bulk materials without the compromise of responsiveness. The liquid-liquid separation process could sequester and concentrate solutes, which facilitated the non-covalent association of building blocks equipped with multiple binding sites and resulted in bulk materials with versatile properties that were highly related to the encapsulated water amounts. It was a robust plastic with a remarkable Young's modulus of $139.53 \pm 4.74 \mathrm{MPa}$ when the water content was about $5 \%$ while became adhesive and could instantly self-heal with more absorbed water. Moreover, the material was reusable and fully recyclable. Our findings suggest that coacervation offers a promising approach to constructing bulk materials using small molecules and new possibilities for the application of supramolecular chemistry.
\end{abstract}




\section{Main}

Plastics are one of the most widely used polymeric materials that are of low cost and high efficient for manufacturing daily products. However, plastics are often difficult to degrade or recycle due to the very stable covalent bonds of macromolecules, resulting in environmental pollution and health problems ${ }^{1}$. To tackle the long-standing challenge to humans from plastics, small molecule-based supramolecular polymers have been proposed to replace conventional polymers ${ }^{2-5}$. Unfortunately, it meets huge challenges to improve the mechanical property and plasticity, impeding applications in practice ${ }^{6,7}$. Such unsatisfactory developments arise from the labile noncovalent bonding between small molecules for supramolecular polymerization. The bond strength of noncovalent interactions is weak, and solvent molecules can interfere with the bonding between solutes, always resulting in a low degree of supramolecular polymerization ${ }^{8,9}$. Keeping these aspects in mind, we propose achieving robust supramolecular plastics by designing small self-assemblers with various potential binding moieties and developing strategies to increase the concentration density of the self-assemblers. Multiple binding sites are beneficial for strengthening the intermolecular association affinity for polymerization through multivalence effects ${ }^{10}$. A high concentration density would reduce the side effects from solvent molecules.

Coacervate droplets are in a dense phase that is separated from a dilute phase at thermodynamic equilibrium ${ }^{11-13}$. Such liquid-liquid phase separation is directed by intermolecular noncovalent interactions, giving rise to stable compartments in solution that sequester and concentrate solutes. In contrast to the formation process of supramolecular materials such as micelles, vesicles and fibres ${ }^{14}$, coacervation can produce larger aggregates - micrometre-scale droplets in solution that can even grow into macroscopic materials consisting of solutes with a high concentration density. Complex coacervation is one of the most studied types of phase separation, highlighting the complexation of oppositely charged macromolecules such as polyelectrolytes ${ }^{15,16}$, peptides and RNAs ${ }^{17-19}$ that drive condensation. They have been widely employed for mimicking biological processes such as surface adhesion ${ }^{20-22}$, cellular compartmentalization $^{23,24}$ and cell communication ${ }^{25,26}$ and exploring advanced applications in the biomedicine ${ }^{27}$ and food industries ${ }^{28}$. However, making coacervates using small building blocks has proven difficult ${ }^{29,30}$.

Here, we demonstrate that charged macrocycles are promising small molecules for producing complex coacervate droplets with countercharged surfactants and complex coacervation can be a powerful strategy for exploring small molecule-based supramolecular plastics with versatile properties. The obtained macroscopic supramolecular material is named a macrocycle-surfactant coacervate (MSC) material. The property of the MSC material was highly related to water content. With a saturated water content, the material was like a fluid and could self-heal instantly even underwater and showed top-of-the-range waterproof adhesiveness in terms of materials made from small molecules. By reducing water molecules, the MSC material (sol) showed a transition to a gel with mechanical properties comparable to those of covalent polymers. Moreover, the photoreversibility and thermoreversibility and simultaneous 
stability equipped the material with excellent processability and usability. Finally, the redox-sensitive disulfide bonds in the macrocycle made the materials biodegradable and fully recyclable. These findings suggest that coacervation represents an effective and powerful strategy for constructing robust bulk supramolecular materials with adhesive properties without compromising the responsiveness and offers an approach for exploring ecofriendly materials that could potentially be substitutes for conventional covalent polymers.

\section{The design of small molecules for complex coacervation}

In our previous work, an azobenzene-derived macrocycle (ADM) linked by disulfide bonds was screened from a dynamic combinatorial library by a highly selective gelation process with magnesium cations $\left(\mathrm{Mg}^{2+}\right)^{31}$. Apart from the electrostatic interaction, the gelation benefited from multiple noncovalent interactions including $\pi$ - $\pi$ stacking between the azobenzene moieties, hydrogen bonding between the amide NH group and carboxylate groups and bridged water interactions between the amide carbonyl or carboxylate group. Encouraged by this successful example of engineering various types of noncovalent interactions in a single molecule to explore supramolecular materials, we regarded ADM as an ideal candidate for coacervation because the repeating units in the macrocycle could multiply the number of noncovalent interactions. Together with ADM, the small cationic surfactant cetyltrimethylammonium bromide (CTAB) was chosen to verify small molecule-based coacervation ${ }^{30}$ (Fig. 1a).

A group of aqueous solutions at $\mathrm{pH}=10$ was prepared from ADM at the same concentration $(10 \mathrm{mM})$ and $\mathbf{C T A B}$ at various concentrations with mole ratios. When the solution was mixed with $\mathbf{A D M}(\mathrm{x} \mathrm{mM})$ and CTAB at a mole ratio of 1:n, the name of the solution was $\mathbf{A D M}(\mathrm{x} \mathbf{m M}) / \mathbf{C T A B} / 1$ :n. The solution $\mathbf{A D M}(10 \mathrm{mM}) / \mathbf{C T A B} / 1: 4$ was homogeneously turbid at the beginning when it was made. Interestingly, a clear and sticky soft material was obtained as a separated phase from the water solution after three hours (Fig. 1b). To gain insights into the formation of the material, time-lapse microscopy was first used to monitor the growth process with time (Fig. 1c-e, Movie S1). In an early stage after mixing, small round droplets with a diameter of approximately $1 \mu \mathrm{m}$ appeared gradually. Thereafter, the diameter of the droplets increased to approximately $20 \mu \mathrm{m}$. Then, the growth kinetics of the droplets prior to full phase separation were investigated using dynamic light scattering (DLS) (Fig. 1f). The sigmoidal profile of the increase in diameter over time was consistent with the typical size growth of coacervates ${ }^{17,25}$.

The coacervation in solutions containing ADM (10 mM) and CTAB at the other ratios was also checked using time-lapse microscopy. MSCs were observed only in the ADM $(10 \mathrm{mM}) / \mathbf{C T A B} / 1: 3$ solution, but their phase separation was not as obvious as that in the ADM $(10 \mathrm{mM}) / \mathbf{C T A B} / 1: 4$ solution (Fig. S1). The zeta potential of the coacervates suggested a neutral charge (Fig. S2). These results suggest that the stoichiometric ratio was the optimized condition for complex coacervation, as it featured a neutral charge stoichiometry, which was consistent with most oppositely charged complex coacervates $^{15,32}$. 

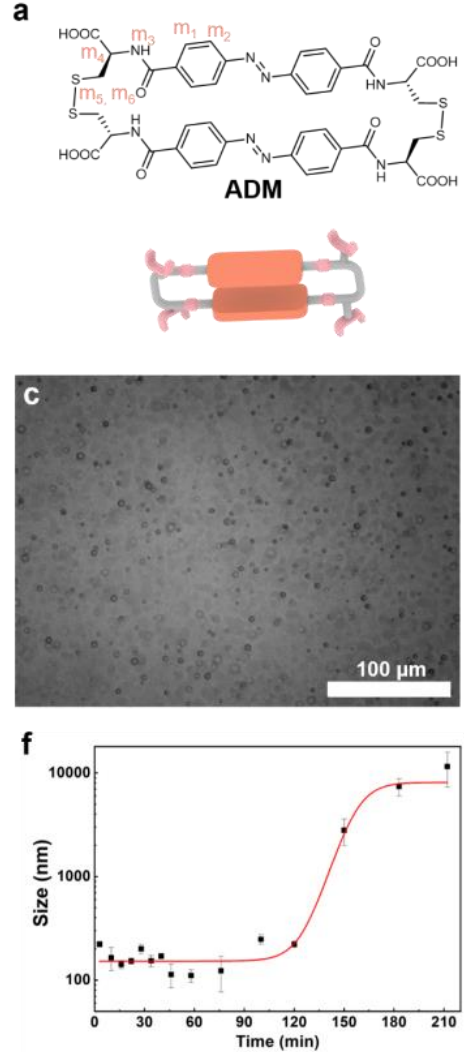

b
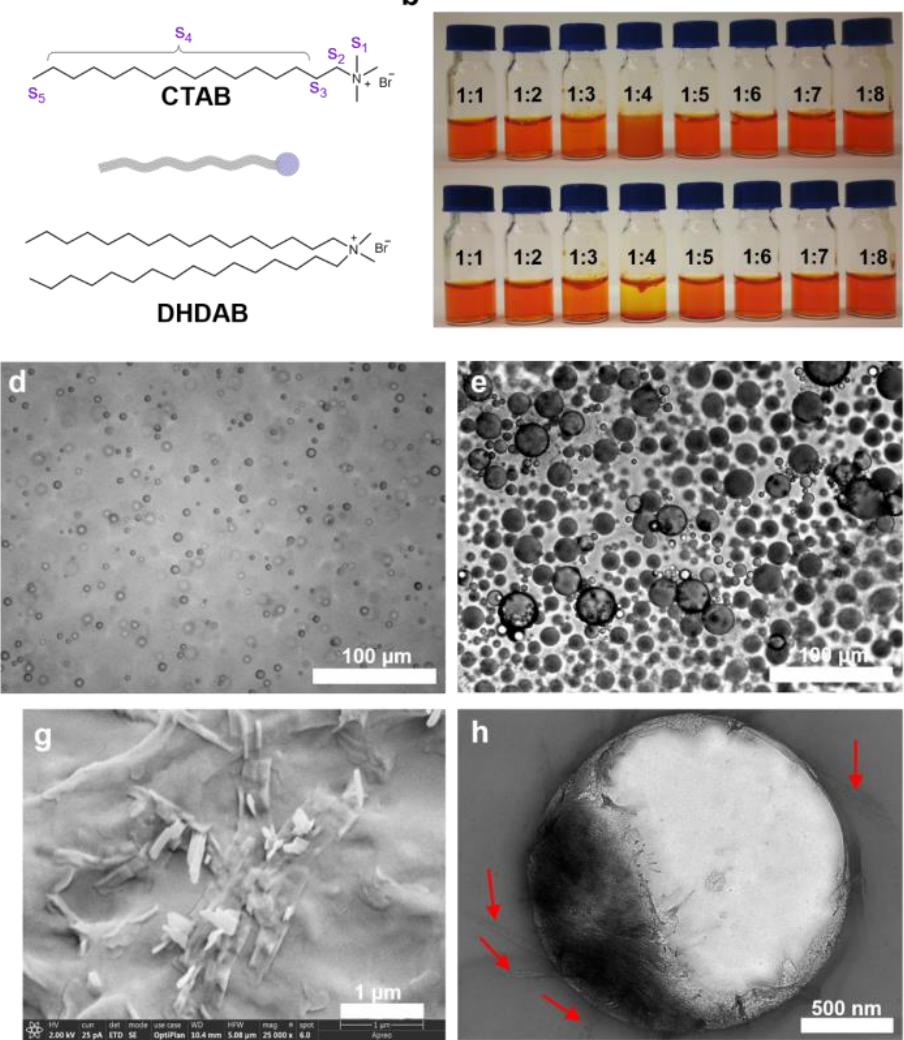

i
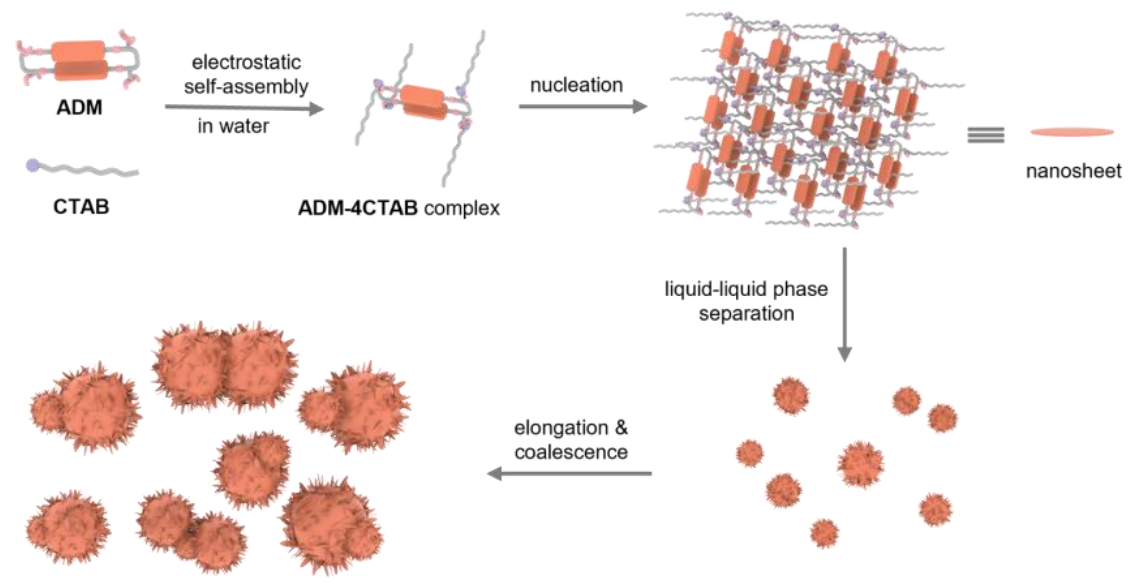

bulk coacervates

droplets

Fig. 1 substructures of coacervate droplets self-assembled by small molecules. a. Chemical structures of ADM macrocycle, CTAB and DHDAB surfactants. $\mathbf{b}$. The appearance of ADM(10 mM)/CTAB/1:n mixture at 3 minutes (top) and 3 hours (bottom) $\left(\mathrm{pH}=\sim 10, \mathrm{H}_{2} \mathrm{O}\right)$ after solution preparation. c-e. Timelapse bright field microscopy images of $\mathbf{A D M}(1 \mathrm{mM}) / \mathbf{C T A B} / 1: 4$ mixture at different time (c: $15 \mathrm{~min}, \mathrm{~d}$ : $30 \mathrm{~min}$, e: $90 \mathrm{~min}$ ) after solution preparation. f. Evolution of the average size of droplets with time before full phase separation in $\mathbf{A D M}(0.1 \mathrm{mM}) / \mathbf{C T A B} / 1: 4$ solution. $\left(\mathrm{pH}=\sim 10, \mathrm{H}_{2} \mathrm{O}\right)$. g. FE-SEM images of bulk ADM-4CTAB MSCs (insert red arrow: nanosheets). $\mathbf{h}$. TEM image of samples taken from ADM(1 $\mathrm{mM}) / \mathbf{C T A B} / 1: 4$ mixture at $15 \mathrm{~min}$ after solution preparation. i. Schematic illustration of mechanism for the self-assembly between ADM macrocycles and CTAB surfactants in water.

\section{Growth of substructures to coacervate droplets}

To determine the microstructures in the MSCs, we lyophilized the condensed phase that 
was isolated from the solution and analysed the morphology of the bulk material using field-emission scanning electronic microscopy (FE-SEM). The surface of the material was attached to nanosheets approximately $100 \mathrm{~nm}$ in width and $500 \mathrm{~nm}$ to $1000 \mathrm{~nm}$ in length, suggesting that the droplets could be organized by the nanosheets (Fig. 1g, S3). This inference was further verified by examining the detailed morphological evolution of the droplets using transmission electron microscopy (TEM). Samples for the analysis were taken at $5 \mathrm{~min}, 15 \mathrm{~min}$ and $120 \mathrm{~min}$ after the solution $(\mathrm{pH} 10)$ ADM $(0.1$ $\mathrm{mM} / \mathbf{C T A B} / 1: 4$ was prepared. Thin nanosheets were observed in the early stage (Fig. S4). Subsequently, small droplets appeared with the nanosheets on their surfaces (Fig. 1h), which was consistent with the FE-SEM analysis. Over time, the small droplets fused into larger droplets with a diameter of approximately $15 \mu \mathrm{m}$, which was similar to the size of droplets observed by time-lapse microscopy at $120 \mathrm{~min}$ after the solution had been made. Thus, we inferred that the three-dimensional coacervate droplets were grown from two-dimensional nanosheets (Fig. 1i).

\section{Molecular assembly behind the complex coacervation}

We proceeded to study the organization of the MSC materials at the molecular level. The composition of the lyophilized material was first identified using proton nuclear magnetic resonance $\left({ }^{1} \mathrm{H} \mathrm{NMR}\right)$ in $\mathrm{CD}_{3} \mathrm{OD}$. The integration of proton signals confirmed that the MSCs were formed by ADM and CTAB with a mole ratio of 1:4 (Fig. S5). The resulting complex was denoted as ADM:4CTAB. Unfortunately, it was not feasible to study the further molecular interaction between the ADM:4CTAB complexes in the ADM/CTAB/1:4 solution using NMR technology owing to the excessively diluted concentration in $\mathrm{D}_{2} \mathrm{O}$ resulting from liquid-liquid phase separation (see Fig. 2a). Noting that solutions prepared from ADM and CTAB with ratios other than 1:3 and 1:4 were homogenous, we first recorded a group of ${ }^{1} \mathrm{H}$ NMR spectra for the solutions ADM(10 $\mathrm{mM}) / \mathbf{C T A B} / 1: \mathrm{n}$ in $\mathrm{D}_{2} \mathrm{O}$ (pD 10) (see Fig. 2a). With the increase of CTAB into the solution of ADM, the peak area of ADM gradually decreased, accompanied by the appearance of new broad peaks at $7.9 \mathrm{ppm}$ and at $7.7 \mathrm{ppm}$ which should be assigned to the protons $m_{1}$ and $m_{2}$ of the azobenzene moiety, respectively. The proton signals $\left(\mathrm{m}_{4}\right.$, $\mathrm{m}_{5}$ and $\mathrm{m}_{6}$ ) of the cysteine moieties in ADM exhibited similar shifting behaviours. All the original sharp peaks of protons at ADM were finally replaced by new broad peaks after the mixing ratio of ADM and CTAB was over 1:4. We assumed that the newly appeared broad peaks were from the aggregates of the complex ADM:4CTAB.

To verify this assumption, we then analysed the solution $\mathbf{A D M}(10 \mathrm{mM}) / \mathbf{C T A B} / 1: 2$ in $\mathrm{D}_{2} \mathrm{O}$ (pD 10) using diffusion ordered spectroscopy (DOSY). The plot of the solution $\mathbf{A D M}(10 \mathrm{mM}) / \mathbf{C T A B} / 1: 2$ in $\mathrm{D}_{2} \mathrm{O}(\mathrm{pD} 10)$ showed only two types of aggregates due to their distinguishable diffusion coefficients ${ }^{33}$. The proton signals $\mathrm{m}_{1}$ ' and $\mathrm{m}_{2}$ ' assigned to the azobenzene moiety shared the same diffusion coefficient $\left(D^{\prime}=9.4842 \times 10^{-11} \mathrm{~m}^{2} / \mathrm{s}\right)$ with protons $\mathrm{s}_{1}, \mathrm{~s}_{2}, \mathrm{~s}_{3}$, and $\mathrm{d}$ from the alkyl chain of $\mathbf{C T A B}$, suggesting that the ADM:4CTAB complex was formed and further piled into large aggregates with higher molecular weights (Fig. 2b). The other type of aggregates were formed by pure ADM, as the protons corresponding to ADM had a diffusion coefficient $\left(D=3.4754 \times 10^{-10}\right.$ $\left.\mathrm{m}^{2} / \mathrm{s}\right)$ similar to that of aggregates in solution prepared from only ADM $(D=$ 
$3.7934 \times 10^{-10} \mathrm{~m}^{2} / \mathrm{s}$ ) (Fig. S6). To understand the interaction between the complex ADM:4CTAB, we also analysed the solutions $\mathbf{A D M}(10.0 \mathrm{mM}) / \mathbf{C T A B} / 1: 2$ and 1:6 in $\mathrm{D}_{2} \mathrm{O}$ (pD 10.0) using two-dimensional nuclear Overhauser effect spectroscopy (NOESY) (Fig. 2c, S7-S8). There were strong nuclear Overhauser effects (NOEs) between protons $\mathrm{m}_{1}$ ' and $\mathrm{m}_{2}$ ' from the newly formed broad peaks of azobenzene moieties and protons s1, s2, s3, and s4 from the alkyl chain of CTAB, while no obvious NOEs were observed between protons $\mathrm{m}_{1}$ and $\mathrm{m}_{2}$ from ADM and the alkyl chain protons of CTAB. This result demonstrated that the alkyl chain of CTAB interpenetrated the ADM macrocycle, giving rise to pseudorotaxane-like substructures for the subsequent formation of supramolecular polymers ${ }^{9,34}$ (Fig. 1i).

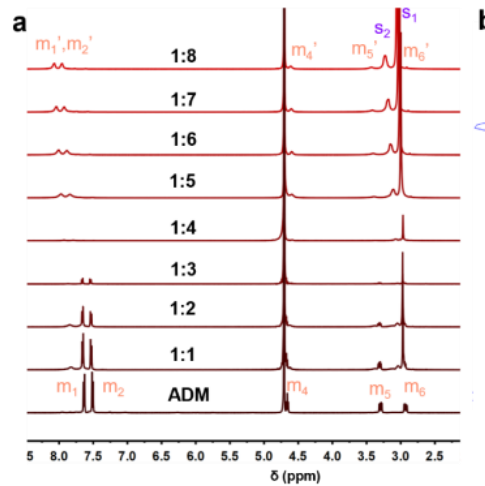

d

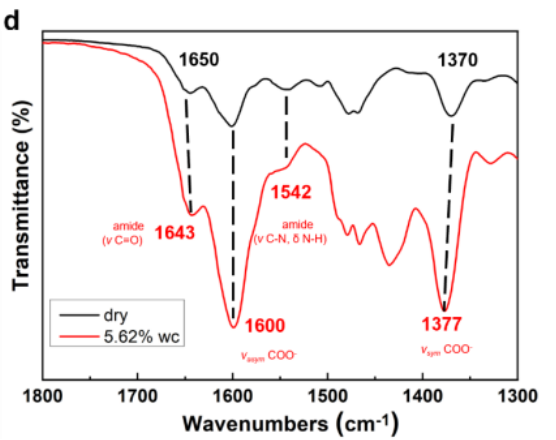

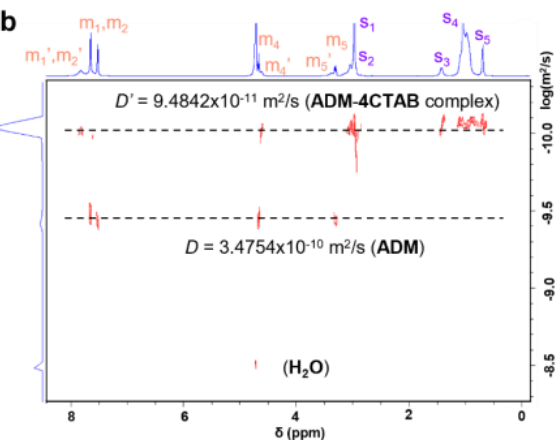

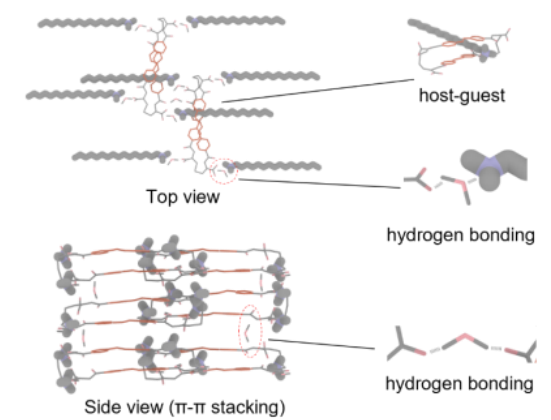

c
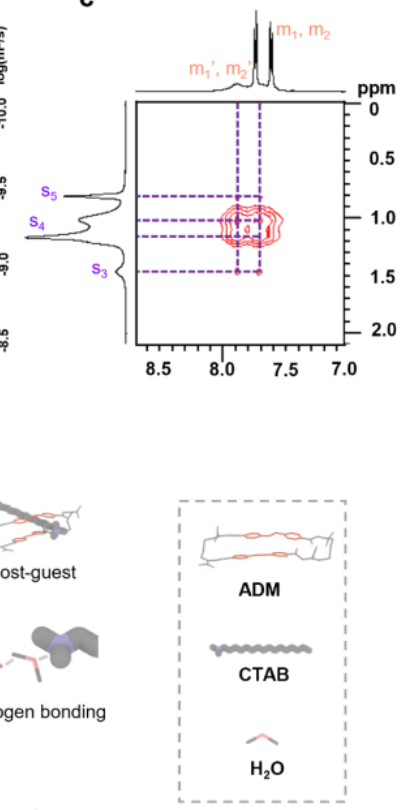

Fig. 2 Molecular assembly behind the complex coacervation. a. Partial ${ }^{1} \mathrm{H}$ NMR spectra of ADM(10 $\mathrm{mM}) / \mathbf{C T A B} / 1: \mathrm{n} .\left(\mathrm{pD}=\sim 10, \mathrm{D}_{2} \mathrm{O}, 500 \mathrm{MHz}\right)$. b. DOSY spectrum of $\mathbf{A D M}(10 \mathrm{mM}) / \mathbf{C T A B} / 1: 2 .(\mathrm{pD}=$ $\left.\sim 10, \mathrm{D}_{2} \mathrm{O}, 600 \mathrm{MHz}\right)$. c. Partial 2D NOESY spectra of ADM(10 mM)/CTAB/1:n. $\left(\mathrm{pD}=\sim 10, \mathrm{D}_{2} \mathrm{O}, 600\right.$ MHz, mixing time $100 \mathrm{~ms}$ ). d. ATR-FTIR spectrum of ADM-4CTAB MSCs in dry (black line) and with $5.62 \%$ water $\left(\mathrm{D}_{2} \mathrm{O}\right)$ content (red line). e. Chemical structure illustration of interwoven ADM4CTAB MSCs in top view and side view, zoom-in pictures show the details of host-guest and hydrogen bonding interaction between carboxylic groups, amide bonds and water molecules.

Previously, we obtained single-crystal structures of ADM coordinated with $\mathrm{Ca}^{2+}$. The spacings of azobenzene units within the ADM and between two adjacent macrocycles were represented by two typical distances of $3.3 \AA$ and $3.6 \AA$, respectively ${ }^{31}$. Indeed, the analysis of the powder XRD data of the lyophilized MSCs (Fig. S9) revealed two distances for $\pi-\pi$ interactions that were $3.98 \AA$ and $4.19 \AA$. The longer distances may be attributed to the interpenetration of the alkyl chain of CTAB into the ADM macrocycle. 
In addition to $\pi-\pi$ interactions, the liquid-liquid separation process for the formation of MSCs cannot take place without the involvement of water molecules ${ }^{13}, 35$. To understand the role of water molecules in the ADM-4CTAB MSCs, we first investigated the hydration and dehydration behaviour of the material (Fig. S10). The maximum percentage of water content in the ADM-4CTAB MSCs was determined to be approximately $45 \%$. The fully hydrated material could be dehydrated in air, and the percentage of water could be minimized to approximately 4\%. Water states of MSCs could be further investigated by thermogravimetric analysis (TGA) and differential scanning calorimeter (DSC) $)^{36-38}$ (Fig. S11-13, Table S1), two distinguished water states including non-freezable (bound) and freezable (free) water could be calculated out (Fig. S13). With the increase of the total amounts of water in the material, the non-freezable water content experienced a linear growth to reach a saturated value around $17 \%$. During the stage of the linear growth, the amounts of the freezable water were negligible, as most of the water was non-freezable. These results indicated that water molecules involved in the formation of the MSCs and may form hydrogen bonding at the hydrophilic sites of the complex ADM:4CTAB.

The role of water molecules in hydrogen bonding was further investigated using attenuated total reflectance-Fourier transform infrared (ATR-FTIR) spectroscopy. ATRFTIR tests were carried out on the ADM-4CTAB MSCs without water and with 5.6\% water content (Fig. 2d, S14). To avoid the overlap of the O-H band of water with the hydrogen bonds in the MSCs, we prepared the samples in deuterated water $\left(\mathrm{D}_{2} \mathrm{O}\right)$ solutions $^{39}$. The initial broad peak at $3368 \mathrm{~cm}^{-1}$ in the ATR-FTIR spectrum of the lyophilized MSC material represents the N-H bond stretching vibration $\left(v_{s} \mathrm{~N}-\mathrm{H}\right)$ of the amide group of ADM. It shifted to $3380 \mathrm{~cm}^{-1}$ in the presence of $\mathrm{D}_{2} \mathrm{O}$, which was attributed to the fact that the addition of $\mathrm{D}_{2} \mathrm{O}$ promoted the gradual exchange of $\mathrm{N}-\mathrm{H}$ bonds to N-D bonds and the formation of hydrogen bonds between $\mathrm{D}_{2} \mathrm{O}$ and $\mathrm{N}-\mathrm{D}^{40}$. In addition, the peak at $1650 \mathrm{~cm}^{-1}$ assigned to the $\mathrm{C}=\mathrm{O}$ stretching of the amid group shifted to $1643 \mathrm{~cm}^{-1}$. This wavenumber downshift was due to hydrogen bonding between the carbonyl group of the amid and $\mathrm{D}_{2} \mathrm{O}$. However, the peak at $1370 \mathrm{~cm}^{-1}$ assigned to the $\mathrm{C}=\mathrm{O}$ symmetric stretching vibration of the carboxylate group was shifted by $+7 \mathrm{~cm}^{-1}$, which was induced by the coordination between the oxygen of $\mathrm{D}_{2} \mathrm{O}$ and the ammonium salt. These results suggested that water molecules bridged interactions between amide carbonyl or carboxylate groups and the ammonium salt, which was similarly observed in the crystal formed by $\mathbf{A D M}$ and $\mathrm{Ca}^{2+20}$.

Taken together, the MSCs were assembled under the direction of multiple noncovalent interactions (Fig. 2e). The MSCs were organized by the ADM:4CTAB complex that was formed through ionic interactions. As the overall charge of the complex was nearly zero, the complex was generally hydrophobic. Due to hydrophobic effects, the alkyl tail of CTAB was buried in the hydrophobic cavity of the ADM macrocycle, resulting in the formation of a pseudorotaxane-like substructure for supramolecular polymerization into two-dimensional structures. In addition, through hydrogen bonding, water molecules bridged the interaction of macrocycles, helping and aligning the $\pi$ - $\pi$ stacking of the azobenzene moieties. The cooperation of these noncovalent interactions led to 
the aggregation of the two-dimensional structure into a dense phase and pushed most water molecules into the diluted phase, giving rise to three-dimensional droplets.

Viscoelastic, self-healing, adhesive and robustness properties of the materials processed from MSCs

We have shown that MSC materials could be reversibly hydrated and dehydrated. By absorbing water, the ADM-4CTAB materials became viscos from elastic. This change was clarified by studying the viscoelasticity of the material with various water contents using a rheometer. Firstly, the transition from a viscoelastic hydrogel to a viscoelastic fluid during the hydration process was confirmed by strain sweep measurements (Fig. 3a-c). All the tested ADM-4CTAB materials had strain ranges where both storage modulus $\left(\mathrm{G}^{\prime}\right)$ and loss modulus $\left(\mathrm{G}^{\prime \prime}\right)$ remained constant, which was defined as straindefined linear viscosity (LVE) regions. In the LVE region, the structure of the sample was not destroyed when the tests were performed. For the material with the water content $\sim 5 \%$, the $\mathrm{G}^{\prime}$ representing elasticity was 10 times larger than the $\mathrm{G}^{\prime \prime}$ representing viscosity in the LVE region, indicating the sample was a typical semi-solid material with a stable gelling state. With the increase of water content to approximately $20 \%$, the $G^{\prime}$ decreased significantly and became nearly identical to $G^{\prime \prime}$, revealing the transition point of semi-solid to semi-fluid. When the water content in the material reached to approximately $45 \%$, the $\mathrm{G}^{\prime}$ was smaller than the $\mathrm{G}^{\prime \prime}$, which was the feature of a viscoelastic fluid.

Considering the dynamics of the non-covalent interactions and molecular conformations for the formation of the ADM-4CTAB materials, we reasoned that the mechanical property should be time-relevant. Then, the viscoelasticity of the ADM4CTAB materials in different time scales were further tested by frequency sweep measurements (Fig. 3d). For the material with a water content approximately 5\%, the $\mathrm{G}^{\prime}$ and $\mathrm{G}^{\prime \prime}$ were not significantly affected by the change of frequency. However, with the increase of water contents in the materials to approximately $20 \%$ and $45 \%$, the both modulis declined as the decrease of frequency while $\mathrm{G}^{\prime}$ was declining in a steeper slope than $G^{\prime \prime}$. This suggested that water molecules enabled the ADM-4CTAB materials to dissipate more energy input in the prolonged timescale. Interestingly, the descending slop was even steeper for the ADM-4CTAB materials with $45 \%$ of water, which could be explained by the sticky rouse polymer dynamics ${ }^{41}$. The additional water enchanced the mobility of the molecular assemblies in the coacervates by competing or dispersing the supramolecular interactions. These results indicated that the water content could effectively regulate the time-relevent viscoelasticity of the ADM-4CTAB material from stable semi-solids to dissipative semi-solids/fluids. 

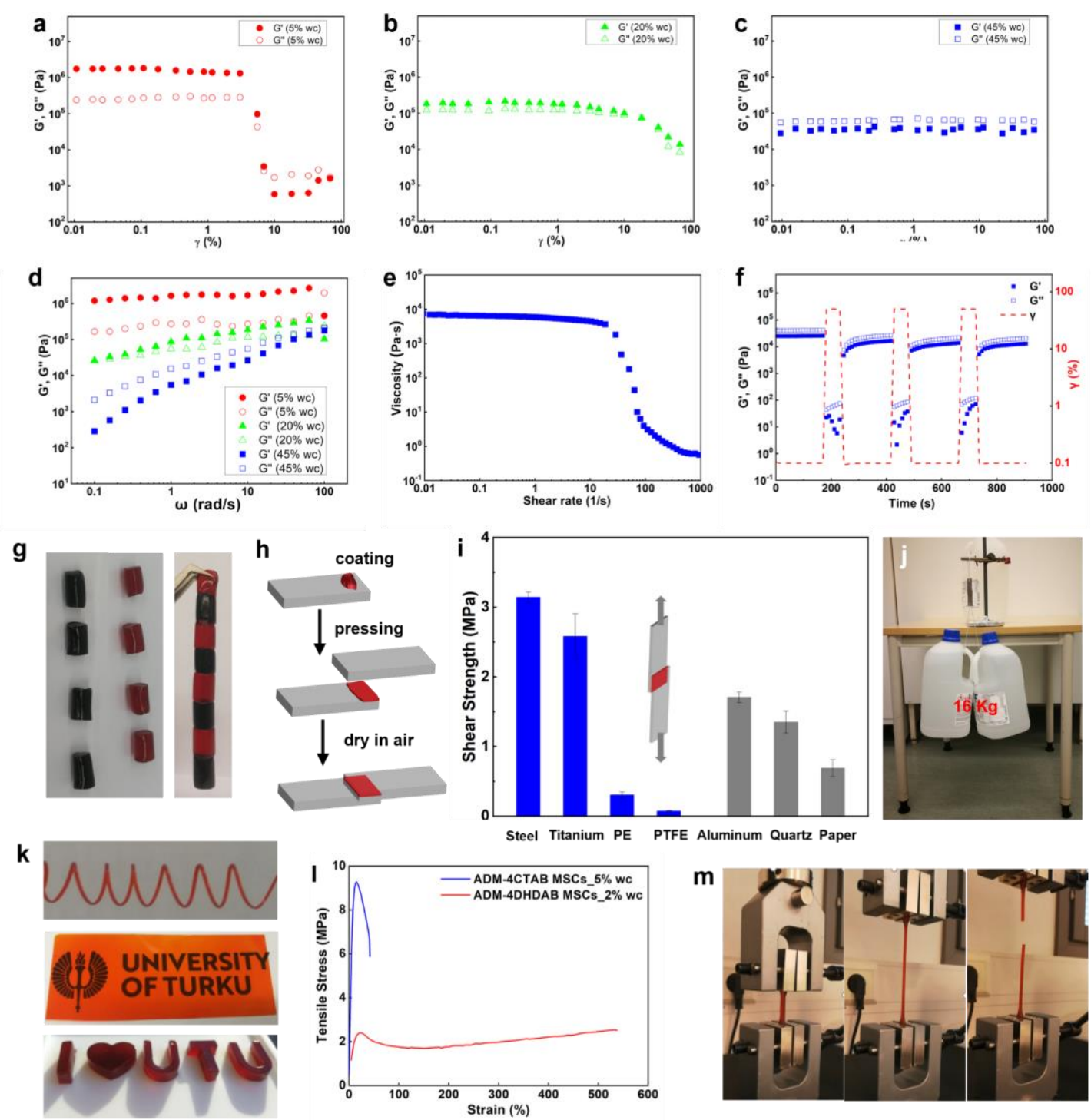

Fig 3. Macro mechanical properties of MSC materials. a-c. Strain sweeps and d. frequency sweeps showing loss ( $\mathrm{G}^{\prime \prime}$, open data point) and storage moduli ( $\mathrm{G}^{\prime}$, filled data points) of bulk ADM-4CTAB MSCs with $\sim 5 \%, \sim 20 \%$ and $\sim 45 \%$ water content. e. Shear-viscosity tests of ADM-4CTAB MSCs with $\sim 45 \%$ water content. f. cyclic strain sweep of ADM-4CTAB MSCs with $\sim 45 \%$ water content. g. Photograph of cylinder-like ADM-4CTAB MSCs specimens after cutting into several fragments and subsequent self-healing process (dark bule specimen was colored by Brilliant blue R-250). h. Brief schematic illustration of lap-shear specimen preparation of ADM-4CTAB MSCs. i. The lap-shear strength of ADM-4CTAB MSCs apply for different substrates (gray bar indicated the substrate plates have been broke before pulled apart). j. Photographs of macroscopic adhesive behavior of ADM-4CTAB MSCs applied to steel substrates. k. Photograph of free-standing ADM-4CTAB MSCs materials after processing into specific shapes (bottom to up: art words, transparent film, spring). l. Tensile-stress curves of free-standing ADM-4CTAB ( 5\% wc) MSCs and ADM-4DHDAB MSCs ( 2\% wc). m. Photograph of free-standing ADM-4DHDAB MSCs after elongation and fracture.

The fluidic properties of the ADM-4CTAB MSCs with saturated water content were further evaluated by checking its dynamic viscosity (Fig. 3e). The viscosity of the material decreased while the shear rate increased, exhibiting shear thinning behaviour. 
This non-Newtonian behaviour usually occurs in solutions of polymers due to the breaking of the physical cross-links between macromolecules under the action of shearing force ${ }^{41,42}$. However, such behaviour shown in the small molecule-based fluidic material suggested that physical cross-linked networks also formed in the MSC material, which probably resulted from the pseudorotaxane substructure. Subsequently, to test the resilience of the physical network, the thixotropy of the material was investigated by cyclic strain sweep (Fig. 3f, S15). The materials were yielded at a strain out of the linear viscoelastic region, showing a dramatic drop of both $\mathrm{G}^{\prime}$ and $\mathrm{G}^{\prime \prime}$. After going back to a strain in the linear viscoelastic region, an instant recovery of materials' strength was shown, which was a common feature of the materials with reversible non-covalent crosslinks. While the recovery was not $100 \%$ reversible in the tested time region, especially for the first cycle. This loss could be attributed to the disentanglement of the molecular assemblies requiring much longer time to reform ${ }^{41}$. Encouraged by the recovery ability of the physical network, we inferred that the material should also be self-healable. Indeed, several pieces of the material cut by a knife automatically selfhealed instantly both underwater and in the air without any other treatment (Fig. 3g, Movie S2). The tensile-stress tests on the self-healed material indicated that $82 \%$ of the fracture strength could be recovered (Fig. S19).

Complex coacervates formed by oppositely charged polyelectrolytes have been proven to be ideal adhesives due to their fluid state, water immiscibility and high density $20-22$, ${ }^{43}$. However, the adhesiveness of small molecule-based coacervates has rarely been studied. Here, the adhesive properties of ADM-4CTAB MSCs containing 45\% water were tested at the interfaces of various substrates (Fig. 3h-3i, S16). Due to the highdensity hydrogen bonding interactions in the ADM-4CTAB MSCs, the material showed high adhesive strength to hydrophilic surfaces, but the strength was weaker on hydrophobic surfaces. The adhesiveness of the material was quantified by determining the shear strength up to $3.14 \pm 0.07 \mathrm{MPa}$ using the specimens with steel substrates. This value was higher than or comparable to most previously reported supramolecular polymer adhesives ${ }^{39,44-46}$. The joint specimens made of steel could hold a $16 \mathrm{~kg}$ weight over a month (Fig. 3j, Movie S3). Furthermore, the adhesive strength could be weakened to $1.57 \pm 0.09 \mathrm{MPa}$ after immersion in water for 12 hours (Fig. S17), but it was still within the top value range for supramolecular adhesives ${ }^{44,47}$.

Finally, the robustness of the dehydrated material was studied. The aforementioned strain sweep measurements of the ADM-4CTAB MSCs containing 5\% water dehydrated from $45 \%$ demonstrated that the material was a hydrogel. A closer inspection of the data revealed that the storage modulus of the material was over a million Pa. In addition, as shown in Fig. 3k, the ADM-4CTAB MSCs with 5\% water content showed ease of processing, as they could be transformed into free-standing objectives with various shapes at room temperature. These results encouraged us to further test the mechanical properties of the material. The tensile-stress test suggested that the ADM-4CTAB MSCs with $\sim 5 \%$ water content had a remarkable Young's modulus, $139.53 \pm 4.74 \mathrm{MPa}$, and a tensile strength of $9.20 \pm 0.11 \mathrm{MPa}$ (Fig. 31, Table S2). However, it showed a maximum fracture elongation of $40 \%$. To improve its 
stretchability, a similar gel was prepared from the same macrocycle ADM and a cationic surfactant dihexadecyldimethylammonium bromide (DHDAB) with two alkyl chains under the same experimental conditions (Fig. 1a). Unlike the aforementioned ADM4CTAB MSCs, the maximum water content of the MSCs made from ADM and DHDAB was only $\sim 18 \%$, and the dehydration curve in air showed that the residue water content was $\sim 2 \%$ (Fig. S18). The elongation at break of the free-standing DHDABbased materials with $2 \%$ water was increased to $528 \%$ (Fig. 31-3m, Movie S4). These results illustrated that the dehydrated MSC material was robust, showing mechanical properties comparable to those of macromolecular polymers ${ }^{48,49}$, even though it was built from small molecules without covalent crosslinkers. By tuning the hydrophobicity of the surfactant, we could control the elongation property of the resulting MSCs.

\section{Stability and responsiveness of the MSCs}

The proven robustness and ease of processing of MSCs should allow us to extend their practical applications as covalent polymers. However, the new materials should be stable yet biodegradable and recyclable. The stability of the material was first evaluated by monitoring the leakage content of the ADM-4CTAB MSCs in distilled water at room temperature and under harsh conditions, i.e., in a solution of phosphate buffered saline (PBS, 1x, pH 7.4) at $37{ }^{\circ} \mathrm{C}$ (Fig. 4a). The material was extremely stable in distilled water but partially dissolved in PBS buffer solution at $37^{\circ} \mathrm{C}$ after 5 days. This was probably because the ionic interaction between the ADM macrocycle and the surfactants was weakened by the buffer salts ${ }^{50}$. However, the stability of the material could be improved by introducing more hydrophobic moieties into the cationic surfactant. The MSC material made from ADM and DHDAB had only $1.5 \%$ leakage into PBS buffer at $37^{\circ} \mathrm{C}$ even after immersion for 15 days. Then, the photostability of the bulk material was tested since the trans form of the azobenzene units in the ADM macrocycle could be isomerized to the cis form under UV light at $365 \mathrm{~nm}$ (Fig. S20), which further induced the disassembly of the ADM-4CTAB coacervate droplets ${ }^{25,51}$ (Fig. 4b-4d). However, no obvious morphological changes of the bulk ADM-4CTAB materials were observed after UV irradiation. Finally, MSC materials are thermosensitive due to the massive hydrogen bonding interactions according to the temperature-variable rheological properties (Fig. S21), but the structure showed good thermostability under heating $\left(100^{\circ} \mathrm{C}\right)$ and cooling cycles $\left(-55^{\circ} \mathrm{C}\right)$ according to the DSC plots (Fig. S12). There were upper-critical solution temperatures (UCST) in the ADM-4CTAB MSCs, which were $\sim 45{ }^{\circ} \mathrm{C}$ and $\sim 27{ }^{\circ} \mathrm{C}$ for the materials with $\sim 5 \%$ and 20\% water contents, respectively (Fig. S21). When bulk ADM-4CTAB MSCs were evaluated by cyclic temperature sweep (Fig. 4e, S22), the phase transition of the material was proven fully reversible. The mechanical modulus could be fully recovered under at least three heating-cooling cycles. The reversible UCST phase transition should be ascribed to the hydrogen bonding and or ionic bonding during the coacervation $^{52}$. 

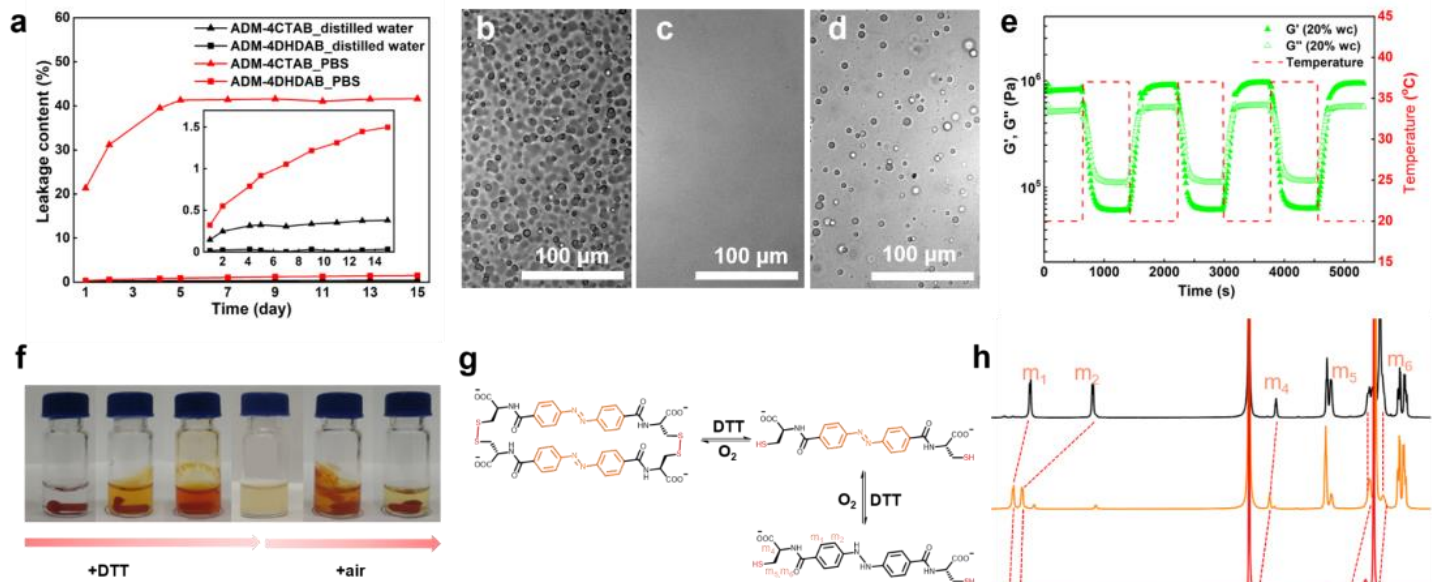

g

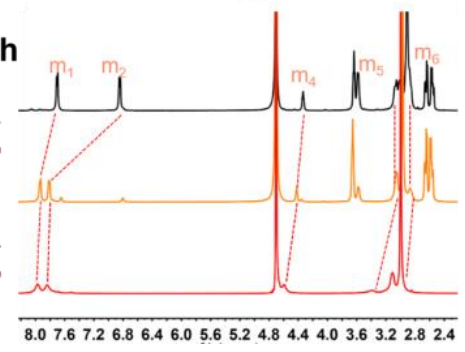

Fig 4. The stability, stimuli responsiveness and recyclability of MSC materials. a. Leakage content of ADM-4CTAB and ADM-4DHDAB MSCs in PBS solution $\left(37^{\circ} \mathrm{C}\right.$, shaker) and distilled water (room temperature). b-d. Bright field microscopy images of ADM-4CTAB MSCs (b) before, (c) after UV 365 $\mathrm{nm}$ irradiation for $60 \mathrm{~s}$, (d) after remove UV light and stay in dark for $5 \mathrm{~min}\left(1 \mathrm{mM}, \mathrm{H}_{2} \mathrm{O}, \mathrm{pH}=10\right.$, room temperature). e. Cyclic temperature sweep measurements of ADM-4CTAB MSCs with 20\% water content. f. The appearance of a piece of bulk ADM-4CTAB MSCs reduction in water by adding $10 \mathrm{eq}$ DTT, and subsequent oxidation by loosening the cap in air. g. The chemical structure illustration of reversible redox of ADM macrocycle by DTT and oxygen. $\mathbf{h}$. The contrastive ${ }^{1} \mathrm{H}$ NMR spectrum of ADM-4CTAB MSCs after adding 10 eq DTT, bottom: before adding DTT (replaced by ADM (10 $\mathrm{mM}) / \mathbf{C T A B} / 1: 5$ aqueous solution), medium: after adding DTT for $5 \mathrm{~min}$, top: after adding DTT for $5 \mathrm{~h}$ $\left(\mathrm{D}_{2} \mathrm{O}, 500 \mathrm{MHz}, 10 \mathrm{mM}\right.$ for $\left.\mathbf{A D M}, \mathrm{pD}=10\right)$.

Apart from the photostability and thermostability of MSCs, the material was biodegradable and fully recyclable. As the ADM macrocycle was synthesized by the linkage of disulfide bonds, it should be redox-responsive. With the addition of a 10 eq. biocompatible reductant dithiothreitol (DTT), a piece of bulk material processed from ADM-4CTAB MSCs, was first degraded into an orange homogeneous aqueous solution in $5 \mathrm{~min}$. Then, the orange solution transformed into a colourless solution after 5 hours (Fig. 4f). We reasoned that the reduction proceeded in two steps by analysing the ${ }^{1} \mathrm{H}$ NMR spectra (Fig. 4g-4h). To facilitate comparative analysis, a homogeneous ADM $(10 \mathrm{mM}) / \mathbf{C T A B} / 1: 5$ aqueous solution was analysed rather than the insoluble ADM-4CTAB MSCs. In the first stage, the disulfide bonds of the ADM macrocycle were reduced to thiol groups ${ }^{29,31}$. The initial wide peaks of protons $m_{1}$ and $m_{2}$ were slightly split into sharp peaks, indicating the disassembly of MSC aggregates. The proton signals of cysteine moieties shifted upfield due to the cleavage of adjacent disulfate. In the second stage, the azobenzene groups were further reduced into diphenylhydrazine groups ${ }^{53}, 54$, accompanied by the fading of solution colour, and protons $\mathrm{m}_{1}$ and $\mathrm{m}_{2}$ near the azobenzene bond significantly shifted upfield. The bulk coacervate material reformed after full oxidation in air overnight (Fig. 4f). Similar to freshly prepared materials, the reformed materials could be easily separated from the solution, and the composition of the recycled material was unchanged, which was 
confirmed by ${ }^{1} \mathrm{H}$ NMR (Fig. S23). In addition, the MSC materials were reusable due to their dynamic supramolecular structures. The MSCs used could be reshaped and reused through a simple process of reabsorbing water at room temperature. The adhesive strength of the material showed no obvious decreases even after reuse many times, and its mechanical properties remained the original strength after recycling many times (Fig. S24). The ${ }^{1} \mathrm{H}$ NMR spectra of recyclable MSC materials further confirmed that the composition remained stable without any spoilage or degradation (Fig. S25).

\section{Discussion}

We have demonstrated that coacervation could be an effective strategy for exploring supramolecular plastics with mechanical properties comparable to those of conventional covalent polymers without the loss of responsiveness. Coacervation was triggered by the ionic interaction between an ionized macrocycle and an oppositely charged surfactant. The properties of the bulk coacervates were highly dependent on the water content inside the material. The initial material separated from the solution retained the most water content and featured fluidic properties. The material had instant self-healing properties and strong adhesive properties in air and underwater. By releasing water, the materials became gels with more solid-like characteristics. The water retention capacity of coacervate materials can be tuned by the hydrophobic proportion of surfactants, which can further exhibit a distinguishable function. Bulk coacervates made from macrocycles ADM and CTAB with a single alkyl tail surfactant showed excellent adhesive strength against hydrophilic substrates at high water contents. When the material was prepared from the same macrocycle and DHDAB with double alkyl tails, the resulting material had a weaker water retention ability but improved robustness and elongation properties. In addition, the MSCs showed highly dynamic and stimuli-responsive properties due to reversible supramolecular interactions and dynamic covalent bonding. The material had fully reversible thermal responsiveness and redox responsiveness and, more significantly, could be reusable and recyclable without obvious performance degradation for many cycles with mild and energy-saving procedures.

In summary, coacervation is usually caused by interactions between polymers. The structural complexity of macromolecules leads to our incomplete understanding of the phase separation phenomenon at the molecular level. Our study of coacervates formed by small molecules with much simpler structures should deepen the knowledge of the molecular interactions that give rise to coacervation. Our findings suggest that small molecule-based coacervation could be applied to construct supramolecular materials that are responsive, biodegradable and fully recyclable, offering new ideas for the application of supramolecular polymers in practical fields in the future, such as recyclable plastics and smart soft materials.

\section{Online content}

Any methods, additional references, Nature Research reporting summaries, source data, extended data, supplementary information, acknowledgements, peer review 
information; details of author contributions and competing interests; and statements of data and code availability are available at XXXXXX.

\section{References}

1. Geyer, R., Jambeck, J. R. \& Law, K. L. Production, use, and fate of all plastics ever made. Sci. Adv. 3, e1700782. (2017).

2. Aida, T., Meijer, E. W. \& Stupp, S. I. Functional supramolecular polymers. Science $\mathbf{3 3 5}$, 813-817. (2012).

3. Yan, X. Z., Wang, F., Zheng, B. \& Huang, F. H. Stimuli-responsive supramolecular polymeric materials. Chem. Soc. Rev. 41, 6042-6065. (2012).

4. Appel, E. A., del Barrio, J., Loh, X. J. \& Scherman, O. A. Supramolecular polymeric hydrogels. Chem. Soc. Rev. 41, 6195-6214. (2012).

5. Harada, A., Takashima, Y. \& Nakahata, M. Supramolecular polymeric materials via cyclodextrin-guest interactions. Acc. Chem. Res. 47, 2128-2140. (2014).

6. Balkenende, D. W. R., Monnier, C. A., Fiore, G. L. \& Weder, C. Optically responsive supramolecular polymer glasses. Nat. Commun. 7, 10995. (2016).

7. Christoff-Tempesta, T. et al. Self-assembly of aramid amphiphiles into ultra-stable nanoribbons and aligned nanoribbon threads. Nat. Nanotechnol. 16, 447-454. (2021).

8. Cafferty, B. J. et al. Efficient self-assembly in water of long noncovalent polymers by nucleobase analogues. J. Am. Chem. Soc. 135, 2447-2450. (2013).

9. Price, T. L. \& Gibson, H. W. Supramolecular pseudorotaxane polymers from biscryptands and bisparaquats. J. Am. Chem. Soc. 140, 4455-4465. (2018).

10. Deng, Y. X., Zhang, Q., Feringa, B. L., Tian, H. \& Qu, D. H. Toughening a self-healable supramolecular polymer by ionic cluster-enhanced iron-carboxylate complexes. Angew. Chem. Int. Ed. 59, 5278-5283. (2020).

11. Michaeli, I., Overbeek, J. T. G. \& Voorn, M. J. Phase separation of polyelectrolyte solutions. J. Polym. Sci. 23, 443-450. (1957).

12. Yewdall, N. A., Andre, A. A. M., Lu, T. M. \& Spruijt, E. Coacervates as models of membraneless organelles. Curr. Opin. Colloid Interface Sci. 52, 101416. (2021).

13. Sing, C. E. \& Perry, S. L. Recent progress in the science of complex coacervation. Soft Matter 16, 2885-2914. (2020). 
14. Sorrenti, A., Illa, O. \& Ortuno, R. M. Amphiphiles in aqueous solution: Well beyond a soap bubble. Chem. Soc. Rev. 42, 8200-8219. (2013).

15. Kizilay, E., Kayitmazer, A. B. \& Dubin, P. L. Complexation and coacervation of polyelectrolytes with oppositely charged colloids. Adv. Colloid Interface Sci. 167, 24-37. (2011).

16. Chang, L. W. et al. Sequence and entropy-based control of complex coacervates. Nat. Commun. 8, 1273. (2017).

17. Aumiller, W. M. \& Keating, C. D. Phosphorylation-mediated RNA/peptide complex coacervation as a model for intracellular liquid organelles. Nat. Chem. 8, 129-137. (2016).

18. Koga, S., Williams, D. S., Perriman, A. W. \& Mann, S. Peptide-nucleotide microdroplets as a step towards a membrane-free protocell model. Nat. Chem. 3, 720-724. (2011).

19. te Brinke, E. et al. Dissipative adaptation in driven self-assembly leading to self-dividing fibrils. Nat. Nanotechnol. 13, 849-855. (2018).

20. Stewart, R. J., Wang, C. S. \& Shao, H. Complex coacervates as a foundation for synthetic underwater adhesives. Adv. Colloid Interface Sci. 167, 85-93. (2011).

21. Zhao, Q. et al. Underwater contact adhesion and microarchitecture in polyelectrolyte complexes actuated by solvent exchange. Nat. Mater. 15, 407-412. (2016).

22. Dompe, M. et al. Thermoresponsive complex coacervate-based underwater adhesive. Adv. Mater. 31, 1808179. (2019).

23. Deng, J. \& Walther, A. Programmable ATP-fueled DNA coacervates by transient liquidliquid phase separation. Chem 6, 3329-3343. (2020).

24. Donau, C. et al. Active coacervate droplets as a model for membraneless organelles and protocells. Nat. Commun. 11, 5167. (2020).

25. Martin, N. et al. Photoswitchable phase separation and oligonucleotide trafficking in DNA coacervate microdroplets. Angew. Chem. Int. Ed. 58, 14594-14598. (2019).

26. Mason, A. F., Buddingh, B. C., Williams, D. S. \& van Hest, J. C. M. Hierarchical self-assembly of a copolymer-stabilized coacervate protocell. J. Am. Chem. Soc. 139, 17309-17312. (2017).

27. Blocher, W. C. \& Perry, S. L. Complex coacervate-based materials for biomedicine. Wiley Interdiscip. Rev.: Nanomed. Nanobiotechnology 9. (2017). 
28. Schmitt, C. \& Turgeon, S. L. Protein/polysaccharide complexes and coacervates in food systems. Adv. Colloid Interface Sci. 167, 63-70. (2011).

29. Abbas, M., Lipinski, W. P., Nakashima, K. K., Huck, W. T. S. \& Spruijt, E. A short peptide synthon for liquid-liquid phase separation. Nat. Chem. 13, 1046-1054. (2021).

30. Douliez, J. P. et al. Catanionic coacervate droplets as a surfactant-based membrane-free protocell model. Angew. Chem. Int. Ed. 56, 13689-13693. (2017).

31. Li, J. W., Cvrtila, I., Colomb-Delsuc, M., Otten, E. \& Otto, S. An "ingredients" approach to functional self-synthesizing materials: A metal-ion-selective, multi-responsive, selfassembled hydrogel. Chem. Eur. J. 20, 15709-15714. (2014).

32. Priftis, D. \& Tirrell, M. Phase behaviour and complex coacervation of aqueous polypeptide solutions. Soft Matter 8, 9396-9405. (2012).

33. Smejkalova, D. \& Piccolo, A. Aggregation and disaggregation of humic supramolecular assemblies by nmr diffusion ordered spectroscopy (DOSY-NMR). Environ. Sci. Technol. 42, 699-706. (2008).

34. Fyfe, M. C. T. \& Stoddart, J. F. Interwoven supramolecular arrays via the noncovalent polymerization of pseudorotaxanes. Coord. Chem. Rev. 183, 139-155. (1999).

35. Zhang, R., Zhang, Y. P., Antila, H. S., Lutkenhaus, J. L. \& Sammalkorpi, M. Role of salt and water in the plasticization of PDAC/PSS polyelectrolyte assemblies. J. Phys. Chem. B 121, 322-333. (2017).

36. Ostrowska-Czubenko, J. \& Gierszewska-Druzynska, M. Effect of ionic crosslinking on the water state in hydrogel chitosan membranes. Carbohydr. Polym. 77, 590-598. (2009).

37. Muller-Plathe, F. Different states of water in hydrogels? Macromolecules 31, 6721-6723. (1998).

38. Ping, Z. H., Nguyen, Q. T., Chen, S. M., Zhou, J. Q. \& Ding, Y. D. States of water in different hydrophilic polymers-DSC and FTIR studies. Polymer 42, 8461-8467. (2001).

39. Dong, S. Y. et al. Structural water as an essential comonomer in supramolecular polymerization. Sci. AdV. 3, eaao0900. (2017).

40. Dzwolak, W., Loksztejn, A. \& Smirnovas, V. New insights into the self-assembly of insulin amyloid fibrils: An H-D exchange FT-IR study. Biochemistry 45, 8143-8151. (2006).

41. Guvendiren, M., Lu, H. D. \& Burdick, J. A. Shear-thinning hydrogels for biomedical applications. Soft Matter 8, 260-272. (2012). 
42. Sun, S. T., Mao, L. B., Lei, Z. Y., Yu, S. H. \& Colfen, H. Hydrogels from amorphous calcium carbonate and polyacrylic acid: Bio-inspired materials for "mineral plastics". Angew. Chem. Int. Ed. 55, 11765-11769. (2016).

43. Narayanan, A., Menefee, J. R., Liu, Q. H., Dhinojwala, A. \& Joy, A. Lower critical solution temperature-driven self-coacervation of nonionic polyester underwater adhesives. ACS Nano 14, 8359-8367. (2020).

44. Shi, C.-Y., Zhang, Q., Tian, H. \& Qu, D.-H. Supramolecular adhesive materials from smallmolecule self-assembly. SmartMat 1, e1012. (2020).

45. Zhao, W. et al. Tunable adhesion from stoichiometry-controlled and sequence-defined supramolecular polymers emerges hierarchically from cyanostar-stabilized anion-anion linkages. J. Am. Chem. Soc. 142, 2579-2591. (2020).

46. Zhang, Q. et al. Exploring a naturally tailored small molecule for stretchable, self-healing, and adhesive supramolecular polymers. Sci. Adv. 4, eaat8192. (2018).

47. Hofman, A. H., van Hees, I. A., Yang, J. \& Kamperman, M. Bioinspired underwater adhesives by using the supramolecular toolbox. Adv. Mater. 30, 1704640. (2018).

48. Sun, T. L. et al. Physical hydrogels composed of polyampholytes demonstrate high toughness and viscoelasticity. Nat. Mater. 12, 932-937. (2013).

49. Yan, X. Z. et al. Quadruple H-bonding cross-linked supramolecular polymeric materials as substrates for stretchable, antitearing, and self-healable thin film electrodes. J. Am. Chem. Soc. 140, 5280-5289. (2018).

50. Priftis, D. et al. Ternary, tunable polyelectrolyte complex fluids driven by complex coacervation. Macromolecules 47, 3076-3085. (2014).

51. Mu, W. J. et al. Membrane-confined liquid-liquid phase separation toward artificial organelles. Sci. Adv. 7, eabf9000. (2021).

52. Erol, O., Pantula, A., Liu, W. \& Gracias, D. H. Transformer hydrogels: A review. Adv. Mater. Technol. 4, 1900043. (2019).

53. Zeyat, G. \& Ruck-Braun, K. Building photoswitchable 3,4'-AMPB peptides: Probing chemical ligation methods with reducible azobenzene thioesters. Beilstein J. Org. Chem. 8, 890-896. (2012).

54. Wong, A. D., Gungor, T. M. \& Gillies, E. R. Multiresponsive azobenzene end-cap for selfimmolative polymers. ACS Macro Lett. 3, 1191-1195. (2014). 


\section{Materials and Methods}

Materials and instruments. Unless otherwise noted, all reagents were purchased from Aldrich and Tokyo Chemical Industry (TCI) and were used as received without further purification. ${ }^{1} \mathrm{H}$ and ${ }^{13} \mathrm{C}$ NMR and 2D NMR, including ${ }^{1} \mathrm{H}-{ }^{1} \mathrm{H}$ COSY, NOESY, and DOSY spectra, were recorded on a Bruker Advance DMX 500 or $600 \mathrm{MHz}$ spectrometer unless otherwise noted. All NMR samples were recorded at $298 \mathrm{~K}$. Mass spectrometry was performed on Finnigan Mat TSQ 7000 instruments. Time-lapse and related bright field microscopy images were recorded by multimode microplate reader equipment with automated digital microscopy and conventional microplate detection (Cyation 5, BioTek Instruments Inc.). Powder X-ray diffraction (XRD) experiments were conducted using an Anton Paar model SAXSess camera equipped with a MicroMax 007 X-ray generator. The sizes and morphologies of the ADM/CTAB complex in solution were observed by using a JEM 1400 plus transmission electron microscope. The samples were dissolved in distilled water, and then $5.0 \mu \mathrm{L}$ of each sample was dropped onto copper grids and air-dried at room temperature. The sizes were measured by using a Zeta sizer Nano-ZS (Malvern Instruments Ltd., Worcestershire, UK) with a He-Ne ion laser of $633 \mathrm{~nm}$, and the $\zeta$-potential was measured with a Zetasizer Nano ZS by using disposable folded capillary cells (DTS1070, Malvern, UK). UV-vis spectra were recorded in a quartz cell (light path $10 \mathrm{~mm}$ ) on a PerkinElmer Lambda $35 \mathrm{UV} / \mathrm{Vis}$ spectrometer. The surface morphologies of ADM-4CTAB MSCs were observed by FE-SEM (Thermo Scientific Apreo S). Both free-standing samples were cut into tiny rectangular shapes with a lancet and dried with a freeze dryer. Both samples were sputter-coated with platinum at a thickness of $5 \mathrm{~nm}$.

Thermal Analysis. DSC was performed on a PerkinElmer Pyris Diamond differential scanning calorimeter. The MSC samples were prepared into thin films, and lyophilized film samples were immersed in distilled water to obtain test samples with different water contents and then encapsulated into a hermetic pan. All the "water content" in the text means the wet basis moisture content (wc) (wc = $\left.\frac{m(\text { wet sample })-m(\text { dry sample })}{m(\text { wet sample })} \times 100 \%\right)$. Each sample was exposed to two heating cycles and one cooling cycle. The heating and cooling rates were both $10 \mathrm{~K} / \mathrm{min}$, and the heating-cooling temperature range was recorded from $218 \mathrm{~K}$ to $373 \mathrm{~K}$. Each test was performed 2 times.

TGA was performed using a PerkinElmer STA 6000 simultaneous thermal analyser, and the heating rate was $20 \mathrm{~K} / \mathrm{min}$ from $303 \mathrm{~K}$ to $1073 \mathrm{~K}$.

ATR-FTIR tests. All MSC samples were prepared in a PTFE mould that was $1 \mathrm{~mm}$ in thickness and 10 $\mathrm{mm}$ in diameter, and a pure DACA sample was formed into powder. Then, the lyophilized bulk MSC plates were immersed in $\mathrm{D}_{2} \mathrm{O}$ to obtain samples with targeted $\mathrm{D}_{2} \mathrm{O}$ content. All the samples were recorded by Fourier transform infrared spectroscopy (FTIR) (Spectrum One, PerkinElmer, Beaconsfield Bucks, UK) with an attenuated total reflectance (ATR) accessory. All the tests were recorded at room temperature.

Rheological tests. All MSC samples were prepared in a PTFE mould that was $1 \mathrm{~mm}$ in thickness and 20 $\mathrm{mm}$ in diameter. Then, the lyophilized bulk MSC plates were immersed in distilled water to obtain test samples with the targeted water contents. The rheological tests were carried out by strain-controlled rheometer (Haake Rheo Stress 300 rheometer) equipped with a parallel plate $20 \mathrm{~mm}$ in diameter (gap, $1.0 \mathrm{~mm}$ ). All the samples were sealed with silicone oil after loading to avoid the evaporation of water. The viscosity of ADM-4CTAB MSCs at $\sim 45 \%$ water content was tested on the same rheometer equipment with a cone plate $20 \mathrm{~mm}$ in diameter and $1^{\circ}$ slope (gap, $0.026 \mathrm{~mm}$ ).

Lap-shear adhesive tests. Lap-shear adhesive specimens were prepared with water-saturated ADM- 
4CTAB MSC materials. Small pieces of materials were picked up with a spoon ( 45\% wc, $30 \mathrm{mg})$, coated evenly on one of the substrates (coating area: $10 \mathrm{~mm} \times 20 \mathrm{~mm}$ ) and covered with another substrate under pressure (400 $\mathrm{g}$ for $15 \mathrm{~min}$ ). Thereafter, the lap-shear specimens were dried at room temperature overnight. The shear strength was tested by material testing machine equipment with two vice grips (model LRX, Lloyd Instrument Ltd, Fareham, England). The tensile-stress curves were recorded with PC-computer software (Nexygen 4.0, Lloyd Instruments Ltd, Fareham, England) until the two lap substrates were separated. The underwater adhesive tests were carried out with the same device, and the specimens were obtained by immersing the dried lap-shear specimens into distilled water for different times before tensile-stress tests. All the strain rates were set to $5 \mathrm{~mm} / \mathrm{min}$.

Tensile-stress tests. All MSC samples were prepared in a dumbbell-like PTFE mould with standard JIS K6251-8 dimensions (length $16 \mathrm{~mm} \times$ width $4 \mathrm{~mm} \times$ thickness $2 \mathrm{~mm}$ ). The lyophilized bulk MSC specimens were dried by a freeze dryer $\left(-50{ }^{\circ} \mathrm{C}, 0.5 \mathrm{mbar}\right.$, ZIRBUS technology, Germany), and freestanding specimens of ADM-4CTAB MSCs at 5\% wc and ADM-4DHDAB MSCs at $2 \%$ wc were obtained after drying in room ambient conditions for 24 hours $\left(\sim 20{ }^{\circ} \mathrm{C}, \mathrm{RH} \sim 30 \%\right)$. All specimens were tested on a material testing machine with two vice grips (model LRX, Lloyd Instrument Ltd, Fareham, England), and the strain-stress curves were recorded with PC-computer software (Nexygen 4.0, Lloyd Instruments Ltd, Fareham, England). Unless otherwise noted, all the strain rates were set to $10 \mathrm{~mm} / \mathrm{min}$. The self-healing specimens were prepared from the original free-standing samples (5\% wc for ADM4CTAB MSCs). First, they were cut into two even pieces in the centre of the dumbbell, then repaired with a drop of distilled water under pressure (400 g) for $15 \mathrm{~min}$, and thereafter dried at room temperature to obtain the test specimens with the targeted water content. The tensile-stress tests were carried out on the same device and parameter settings as the original tests.

\section{Data availability}

Data generated and analysed during this study are provided as source data with this paper or included in the Supplementary Information. Further data are available from the corresponding authors upon request.

\section{Acknowledgements}

We are grateful for the financial support from the Sigrid Jusélius Foundation and the Academy of Finland (Decision No.318524). TEM imaging was performed in the Laboratory of Electron Microscopy, Institute of Biomedicine, the University of Turku, which receives financial support from Biocentre Finland. We thank Mr. C. Chen for performing preliminary experiments. We are grateful for Prof. S. Otto (University of Groningen, the Netherlands) for providing us the ADM for initial tests and Prof. D. Adams (University of Glasgow, GB) for his insightful comments to the material.

\section{Author contributions}

J.Y. and J.L. conceived the idea and designed the experiments. J.Y. executed most of the experiments and analysed the data. D.Q. designed the rheological characterizations and helped the experiments. E.M. helped the FE-SEM, TGA and DSC measurement. L.L. and P.V. helped the ATR-FTIR tests and other mechanical tests. A.P., M.P., D.Q., J.R., S.J. and C.J. contributed to the measurement of powder XRD, TEM and DLS and monitoring the coacervation process, respectively. J.Y., Z.Z. and J.L. wrote the paper. All authors discussed the experiments, edited the manuscript and gave consent for this publication under the supervision of J.L. 


\section{Supramolecular Plastics Processed from Small Molecule-based}

\section{Coacervates}

Jingjing Yu ${ }^{1}$, Dawei Qi ${ }^{1}$, Ermei Mäkilä2 ${ }^{2}$ Lippo Lassila ${ }^{3}$, Anastassios C. Papageorgiou ${ }^{4,5}$, Markus Peurla $^{6}$, Jessica M. Rosenholm ${ }^{7}$, Zhao Zhao ${ }^{1}$, Pekka Vallittu ${ }^{3,8}$, Sirpa Jalkanen ${ }^{1}$, Chunman Jia ${ }^{9,10}$, Jianwei $\mathrm{Li}^{1,9,10 *}$

${ }^{I}$ MediCity Research Laboratory, University of Turku, Tykistökatu 6, 20520 Turku, Finland

${ }^{2}$ Laboratory of Industrial Physics, Department of Physics and Astronomy, University of Turku, 20014 Turku, Finland

${ }^{3}$ Department of Biomaterials Science and Turku Clinical Biomaterials Centre - TCBC, Institute of Dentistry, University of Turku, 20014 Turku, Finland

${ }^{4}$ Turku Bioscience Centre, University of Turku, 20521 Turku, Finland

${ }^{5}$ Turku Bioscience Centre, Åbo Akademi University, 20521 Turku, Finland

${ }^{6}$ Institute of Biomedicine and FICAN West Cancer Research Laboratories, University of Turku, 20014 Turku, Finland

${ }^{7}$ Pharmaceutical Sciences Laboratory, Faculty of Science and Engineering, Åbo Akademi University, Tykistökatu 6, 20520 Turku, Finland

${ }^{8}$ City of Turku Welfare Division, Puolalankatu 5, 20101 Turku, Finland

${ }^{9}$ Hainan Provincial Key Laboratory of Fine Chem, School of Chemical Engineering and Technology, Hainan University, 570228 Haikou, China.

${ }^{10}$ One Health Institute, Hainan University, 570228 Haikou, China.

* Corresponding author, e-mail: Jianwei.li@utu.fi

\section{Table of contents}

1. Synthetic protocols of compounds S1

2. Preparation of bulk ADM-4CTAB and ADM-4DHDAB MSCs materials ............................. S1

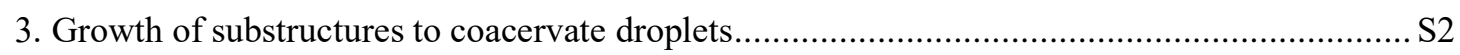

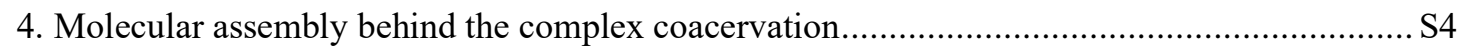

5. Self-healing, adhesive and robust properties of materials processed from the MSCs ............. S10

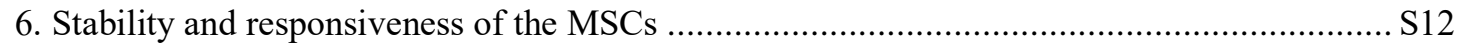

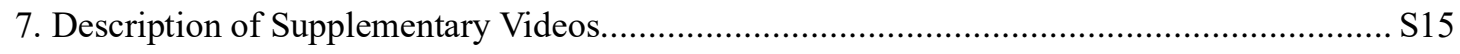

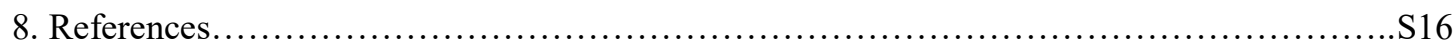




\section{Synthetic protocols of compounds}
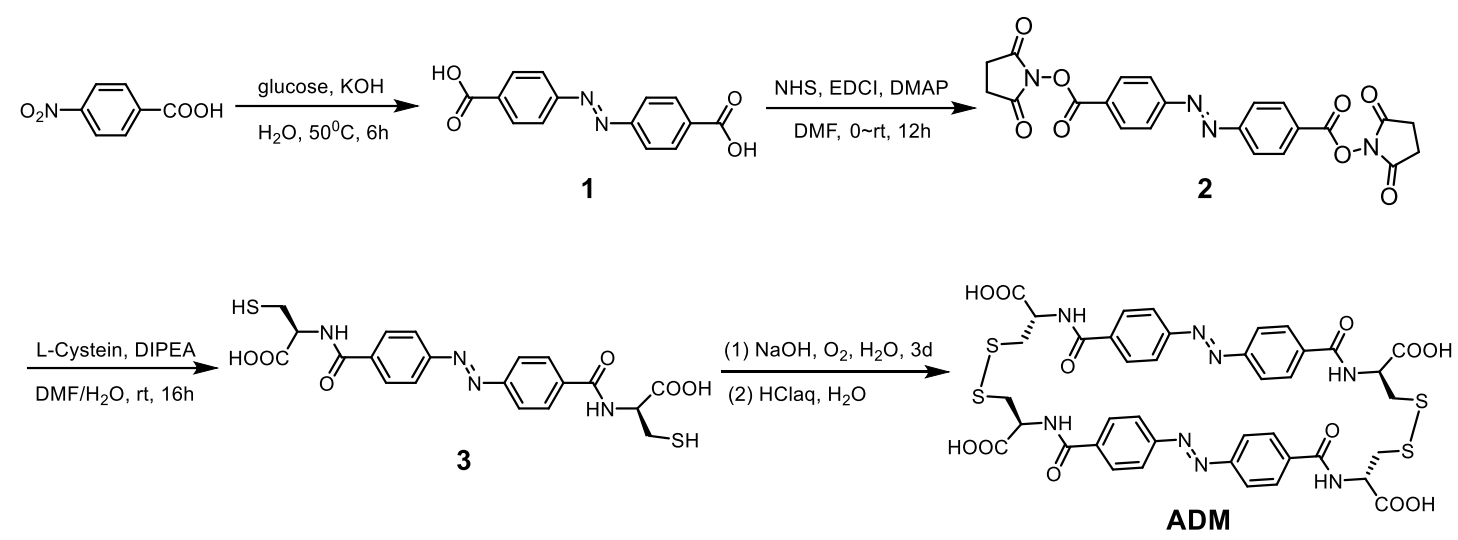

Scheme S1. The synthetic route of ADM macrocycle.

N-hydroxy succinimide (NHS) (10.23 g, $88.8 \mathrm{mmol}$ ) was added to a dimethylformamide (DMF) solution of azobenzene-4,4'-dicarboxylic acid $(10.00 \mathrm{~g}, 37.0 \mathrm{mmol})$, and the solution was placed into an

ice bath. Catalytic 4-dimethylaminopyridine (DMAP) and 1-ethyl-3-(3dimethylaminopropyl)carbodiimide (EDCI) (17.02 g, $88.8 \mathrm{mmol})$ were added successively. After that, the reaction solution was placed in a $60{ }^{\circ} \mathrm{C}$ oil bath and stirred for another 12 hours under a nitrogen atmosphere. Thereafter, the reaction solution was cooled to room temperature, and $500 \mathrm{~mL}$ water was added. Massive precipitates appeared, and the turbid solution was filtered with a Buchner funnel and washed with water three times. Product 2 was obtained as a solid $(15.5 \mathrm{~g})$ with $90 \%$ yield without further purification.

Compound 2 ( $5 \mathrm{~g}, 10.8 \mathrm{mmol}$ ) was dissolved in DMF, aqueous L-cysteine (3.26 g, $26.9 \mathrm{mmol}, 50$ $\mathrm{ml})$ and $N, N$-diisopropylethylamine (DIPEA) $(5.57 \mathrm{~g}, 43.1 \mathrm{mmol})$. The reaction solution was stirred at room temperature overnight. The reaction terminus was confirmed by thin layer chromatography (TLC). After that, most of the solvent was removed by a vacuum rotary evaporator at $60^{\circ} \mathrm{C}$ and then poured into $1 \mathrm{~L}$ water, and massive precipitates appeared. The turbid solution was filtered with a Buchner funnel and washed with water three times.

Then, the precipitates were redissolved in water after adding $\mathrm{NaOH}(4.31 \mathrm{~g}, 107.7 \mathrm{mmol})$. The solution was bubbled with an air pump for three days. After that, the $\mathrm{pH}$ of the reaction solution was adjusted to be acidic $(\sim 2)$, massive precipitates were removed, and the turbid solution was filtered with a Buchner funnel and washed with water three times. The precipitates were dried by a freeze dryer without further purification. The final product was an orange powder $(3.83 \mathrm{~g})$ with $\sim 75 \%$ yield in a total of two steps. ${ }^{1} \mathrm{H}$ NMR $\left(500 \mathrm{MHz}, \mathrm{D}_{2} \mathrm{O}, 298 \mathrm{~K}\right) \delta(\mathrm{ppm}): 7.74(\mathrm{~d}, J=8.5 \mathrm{~Hz}, 8 \mathrm{H}), 7.62(\mathrm{~d}, J=8.5 \mathrm{~Hz}, 8 \mathrm{H})$, $4.76(\mathrm{~m}, 4 \mathrm{H}), 3.39$ (dd, $J=13.8,6.5 \mathrm{~Hz}, 4 \mathrm{H}), 3.03(\mathrm{dd}, J=13.8,7.7 \mathrm{~Hz}, 4 \mathrm{H}) .{ }^{13} \mathrm{C} \mathrm{NMR}\left(125 \mathrm{MHz}, \mathrm{D}_{2} \mathrm{O}\right.$, $298 \mathrm{~K}) \delta$ (ppm): 176.75, 168.36, 152.93, 135.34, 128.26, 122.79, 55.31, 40.66. HR-MS (ESI) (m/z): [M $+\mathrm{H}]^{+}$calcd for $\mathrm{C}_{29} \mathrm{H}_{18} \mathrm{~F}_{6} \mathrm{O}_{8} \mathrm{~S}_{2} 949.1408$, found 949.1442 .

\section{Preparation of bulk ADM-4CTAB and ADM-4DHDAB MSCs materials}

ADM-4CTAB MSCs. First, ADM powder $(1.00 \mathrm{~g}, 1.05 \mathrm{mmol})$ was dissolved in $\mathrm{NaOH}$ aqueous solution $(\mathrm{pH}=10,50 \mathrm{ml})$, and $\mathrm{CTAB}(1.54 \mathrm{~g}, 4.20 \mathrm{mmol})$ was dissolved in distilled water $(50 \mathrm{ml})$. Basic ADM solution was added dropwise to the CTAB aqueous solution at room temperature under stirring. Several minutes later, the mixing solution became turbid, and massive 
red sticky materials precipitated after ageing for 3 hours. The materials were separated from the solution by centrifugation, and then the separated bulk ADM MSCs were placed directly into distilled water for dialysis for 3 days to remove residual unbound components. The final pure ADM MSCs were be obtained at approximately $85 \%$ yield.

ADM-4DHDAB MSCs. DACA powder $(1.00 \mathrm{~g}, 1.05 \mathrm{mmol})$ was dissolved in $\mathrm{NaOH}$ aqueous solution $(\mathrm{pH}=10,50 \mathrm{ml})$, and more hydrophobic DHDAB $(2.41 \mathrm{~g}, 4.20 \mathrm{mmol})$ was added to 500 $\mathrm{ml}$ distilled water at $50{ }^{\circ} \mathrm{C}$ to obtain a colloidal solution. Then, the ADM basic solution was added dropwise at room temperature under stirring. Several minutes later, massive red flocculent materials precipitated out, and the mixing solution was aged for 3 hours to reach equilibrium. The flocculent materials were further filtered with a stainless sifter and processed into bulk with pressure. Then, the separated bulk ADM-4DHDAB MSCs were placed directly into distilled water for dialysis for 3 days to remove residual unbound components. The final pure ADM-4DHDAB MSCs could be obtained in approximately $92 \%$ yield.

Considering that bulk MSC materials formed from liquid-liquid phase separation in a short time, the formed materials were not uniform. Herein, a simple strategy was applied to obtain more homogenous bulk materials ${ }^{1}$. Water-saturated bulk MSC materials were first fully dissolved into a glass petri dish with a good solvent (methanol or ethanol) and then placed into a glass petri dish in a fume hood to remove volatile methanol. A transparent and thin film of MSCs could be obtained after it was peeled from the glass surface. The residual methanol was removed after reimmersing the films into distilled water overnight. The final bulk materials could be processed into different shapes on demand.

\section{Growth of substructures to coacervate droplets}

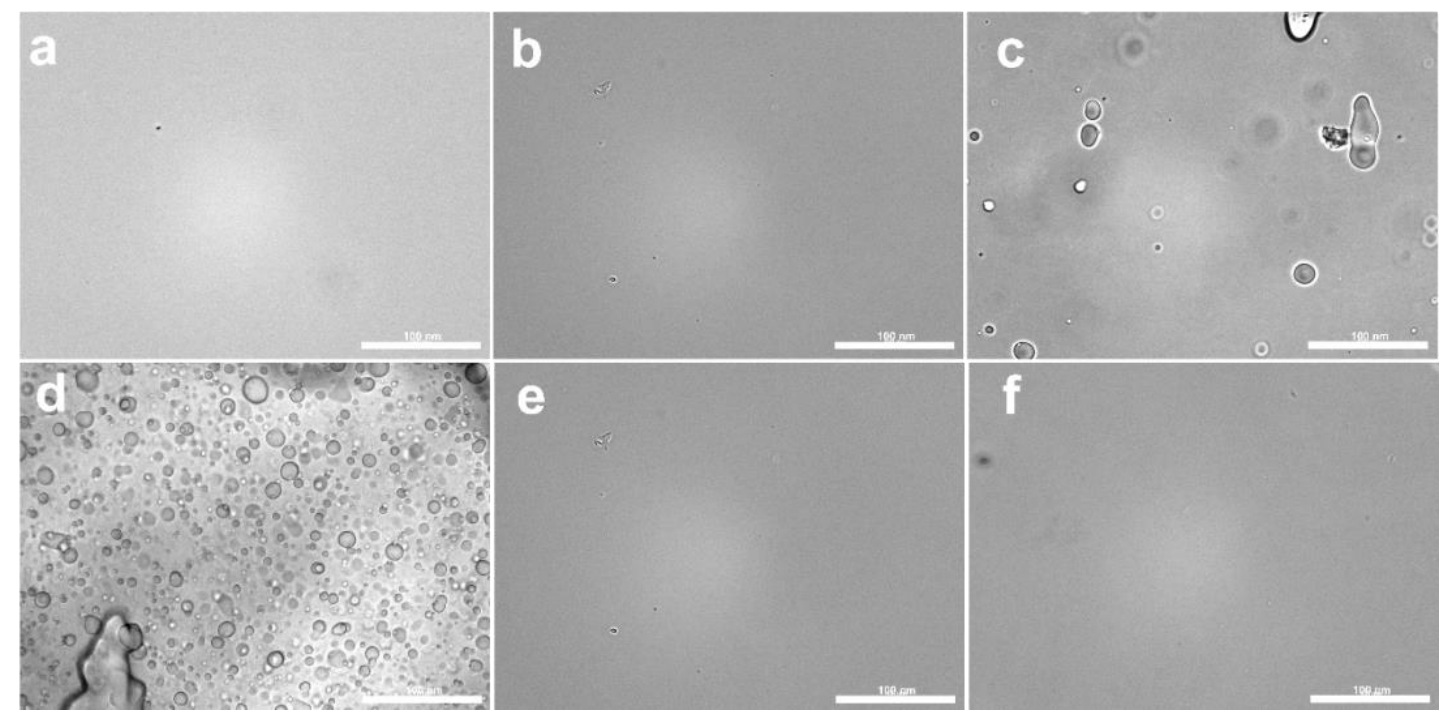

Fig. S1. The microscopy images of ADM(1 mM)/CTAB/1:n (a-f, 1:1-1:6) complex solution after mixing for $30 \mathrm{~min}$. $\left(\mathrm{pH}=10, \mathrm{H}_{2} \mathrm{O}\right.$, scale bar $\left.100 \mathrm{~nm}\right)$. 


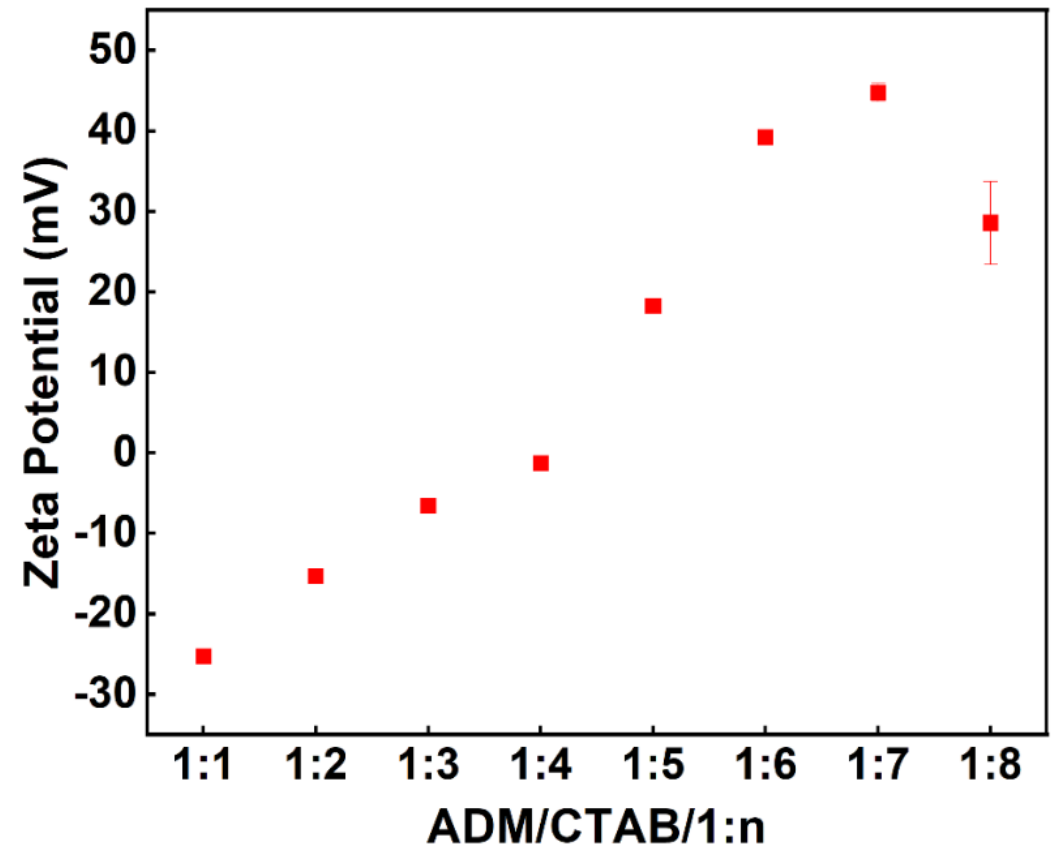

Fig. S2. The zeta potential of $\mathbf{A D M}(0.1 \mathrm{mM}) / \mathbf{C T A B} / 1: \mathrm{n}$ aqueous solution after mixing for $3 \mathrm{~h}$ $(\mathrm{pH}=10)$.
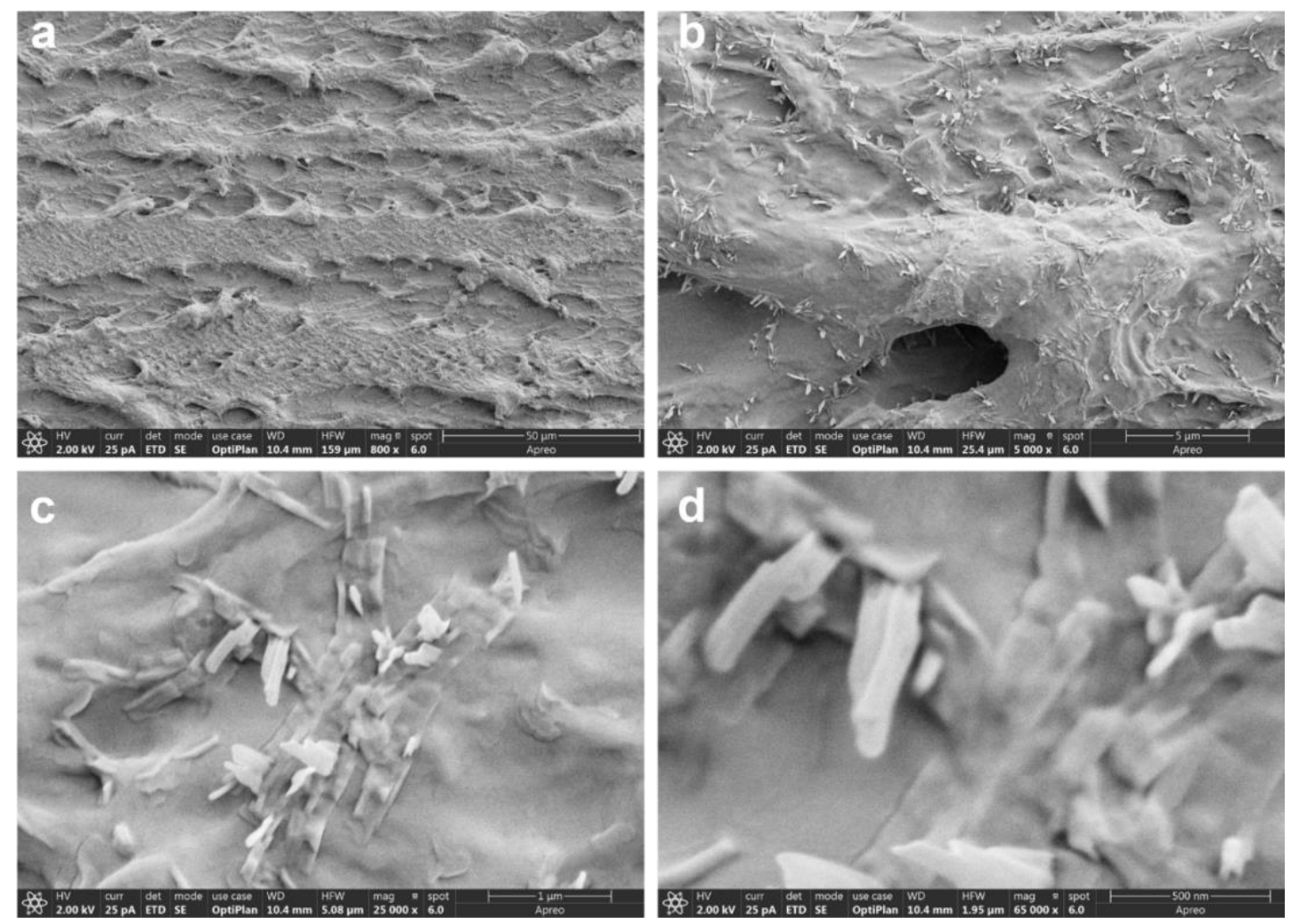

Fig. S3 FE-SEM images of bulk ADM-4CTAB MSCs at different scale bar. 

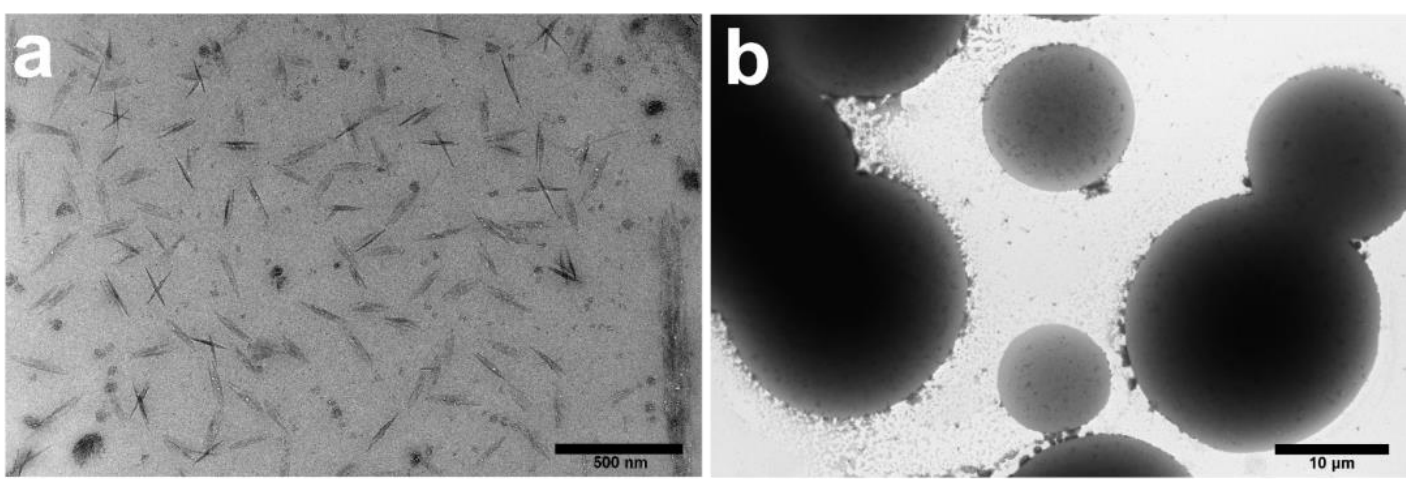

Fig. S4 TEM images of ADM(1 mM)/CTAB/1:4 after for (a) 5 min and (b) $120 \mathrm{~min}$.

\section{Molecular assembly behind the complex coacervation}

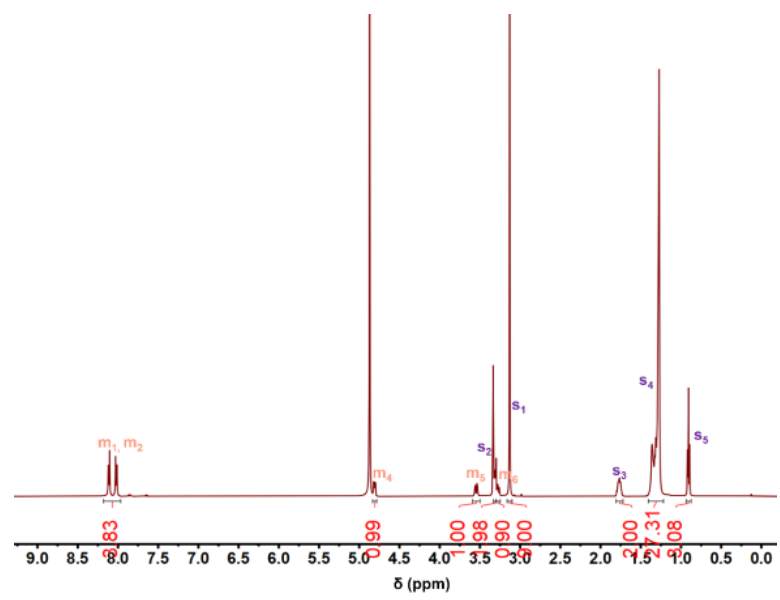

Fig. S5 ${ }^{1} \mathrm{H}$ NMR of lyophilic ADM-4CTAB $\left(\mathrm{CD}_{3} \mathrm{OD}-\mathrm{d}^{4}, 500 \mathrm{MHz}, 298 \mathrm{~K}\right)$

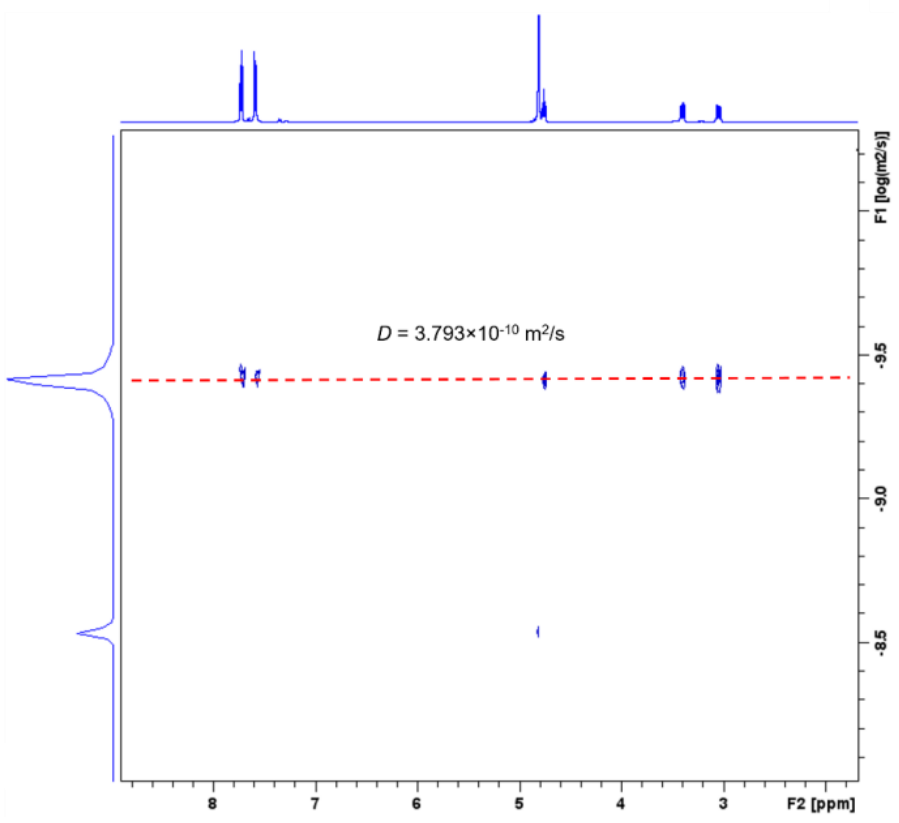

Fig. S6. The 2D DOSY spectra of ADM macrocycle. (10 mM, $\left.\mathrm{D}_{2} \mathrm{O}, \mathrm{pD}=10,600 \mathrm{MHz}, 298 \mathrm{~K}\right)$ 


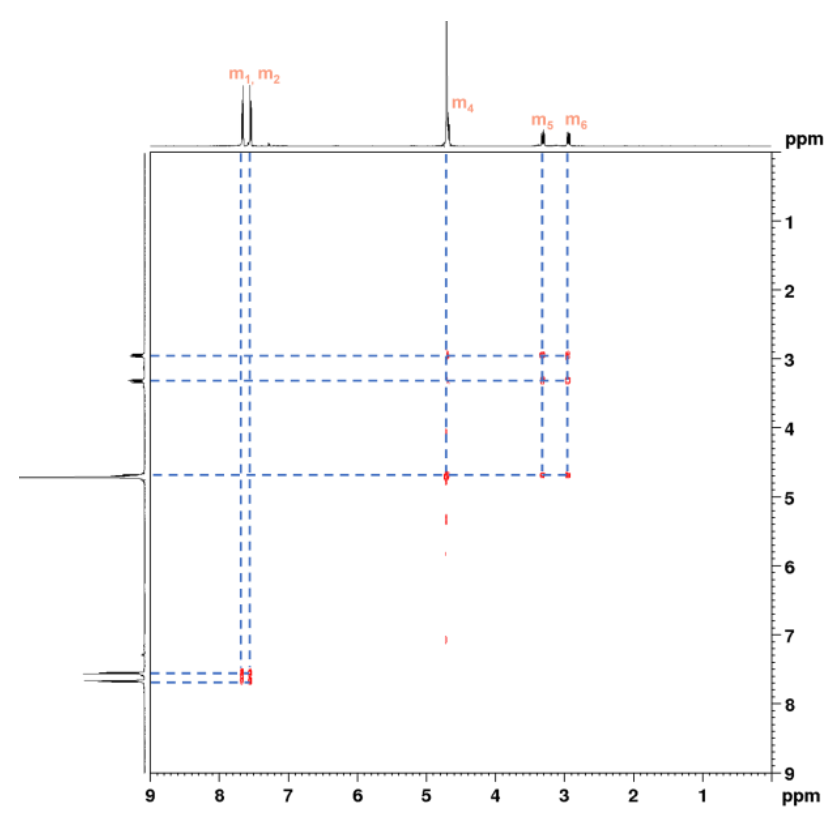

Fig. S7 2D ${ }^{1} \mathrm{H}-{ }^{1} \mathrm{H}$ COSY spectra of ADM $\left(10 \mathrm{mM}, \mathrm{pD}=10, \mathrm{D}_{2} \mathrm{O}, 600 \mathrm{MHz}\right)$
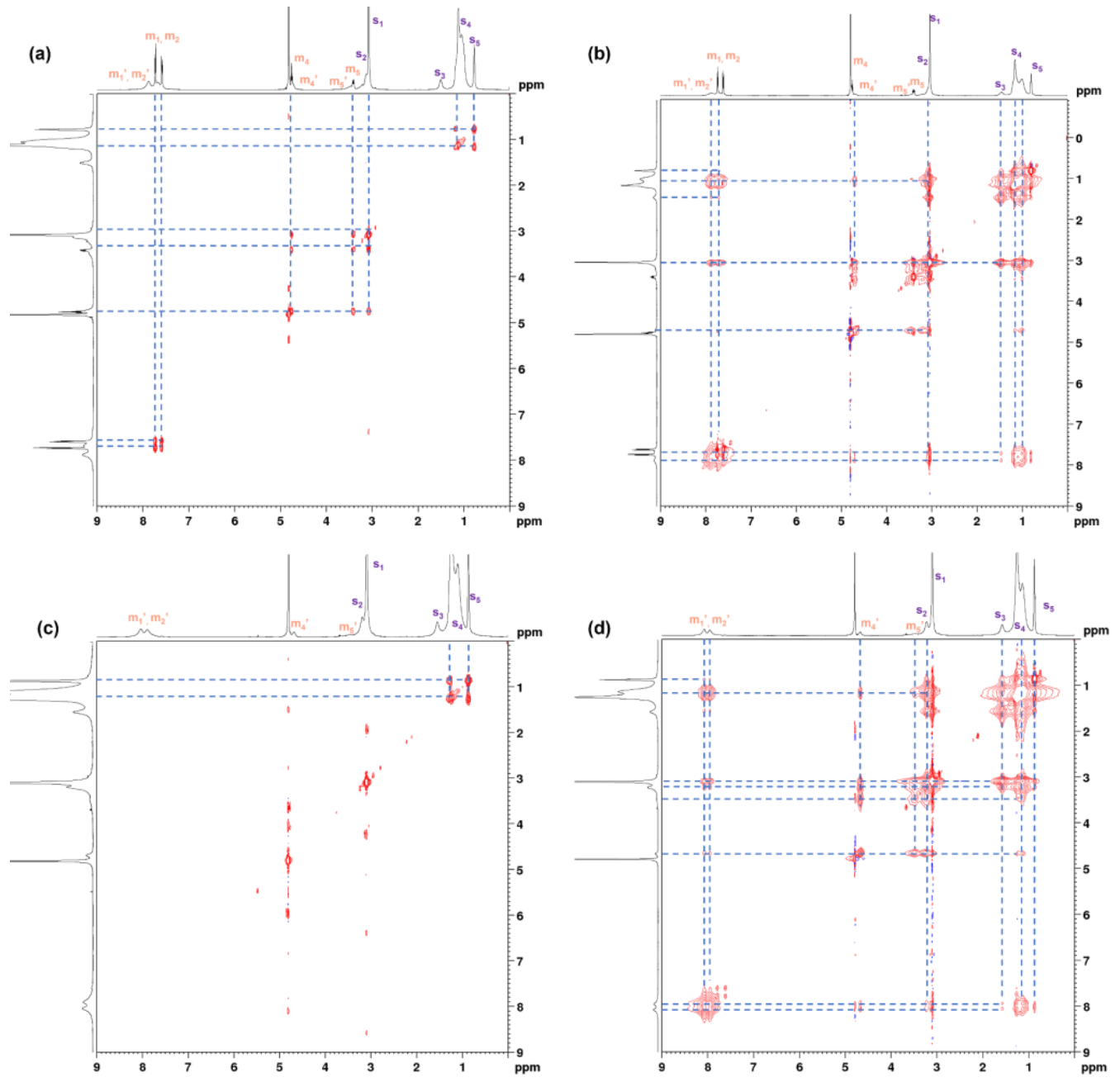

Fig. S8 2D ${ }^{1} \mathrm{H}^{1} \mathrm{H}$ COSY spectrum of (a) $\mathbf{A D M}(10 \mathrm{mM}) / \mathbf{C T A B} / 1: 2$ and (c) $\mathbf{A D M}(10$ mM)/CTAB/1:6. 2D ${ }^{1} \mathrm{H}-{ }^{1} \mathrm{H}$ NOESY spectrum of (b) ADM(10 mM)/CTAB/1:2 and (d) ADM(10 $\mathrm{mM}) / \mathbf{C T A B} / 1: 6 .\left(\mathrm{pD}=10, \mathrm{D}_{2} \mathrm{O}, 600 \mathrm{MHz}\right.$, mixing time $\left.=100 \mathrm{~ms}\right)$. 


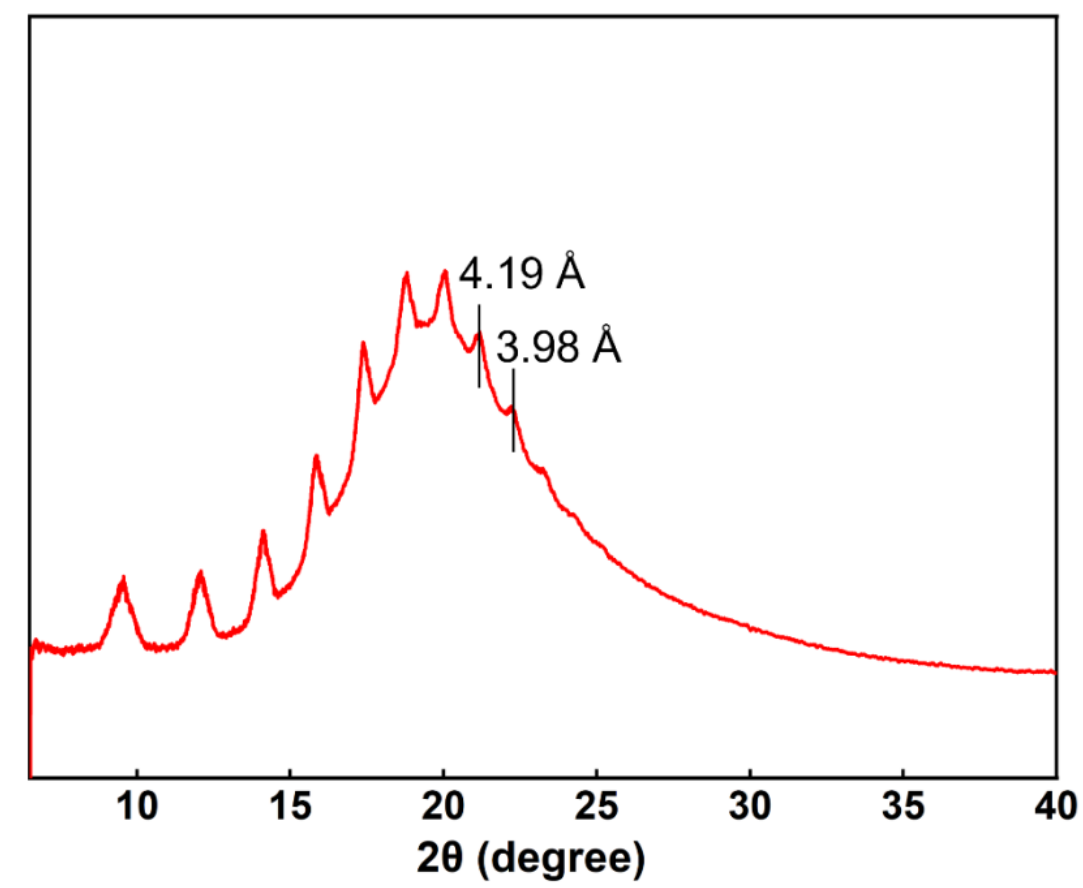

Fig. S9 PXRD of lyophilic bulk ADM-4CTAB MSCs.

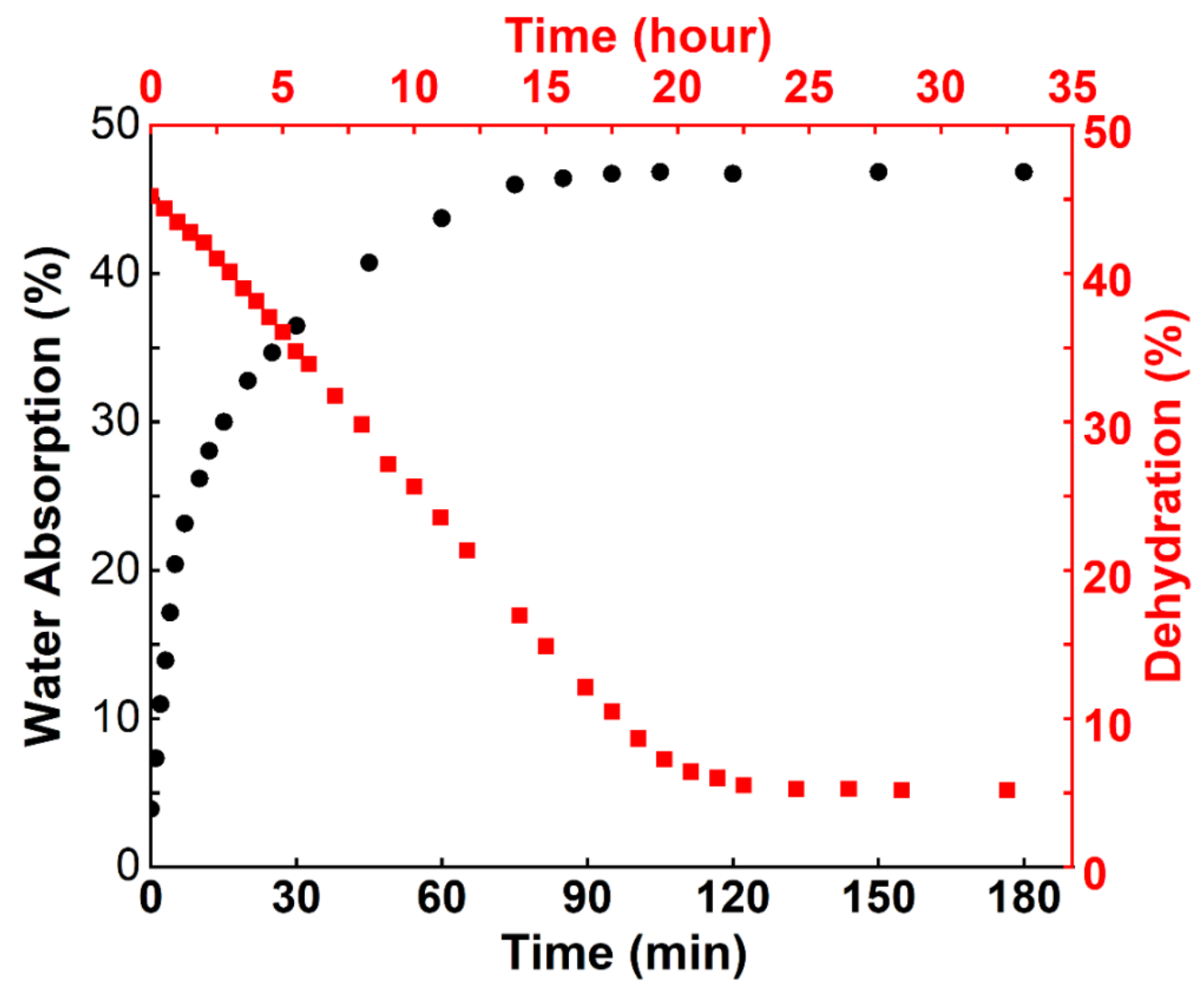

Fig. S10. The water absorption and dehydration kinetic curves of ADM-4CTAB MSCs. 
It is well-known that the absorbed water molecules in a polymeric material either stay proximity (bind) to the hydrophilic surface of macromolecules or far away. As the bounded water molecules have lower energy than the free water molecules, their freezing point is depressed and lower. Thus, these two types of water are also called non-freezable water and freezable. It is of strong interest to investigate the non-freezable water, as it is tightly bound at the hydrophilic site of the polymer and thereby has significant impacts on the structure and property of the resulting material.

The different states of water inside MSCs could be first analyzed by TGA methods. The TGA curves of ADM-4CTAB MSCs with different water content were shown in Fig. S11. In general, the MSC materials showed good stability under $200{ }^{\circ} \mathrm{C}$, no obvious weight-losing was observed except water evaporation. However, the evaporated rates of the samples were different. The original fast evaporated rate belonged to free water and the later slower rate belonged to bound water ${ }^{2}$, which suggested the different water states inside the MSC materials.

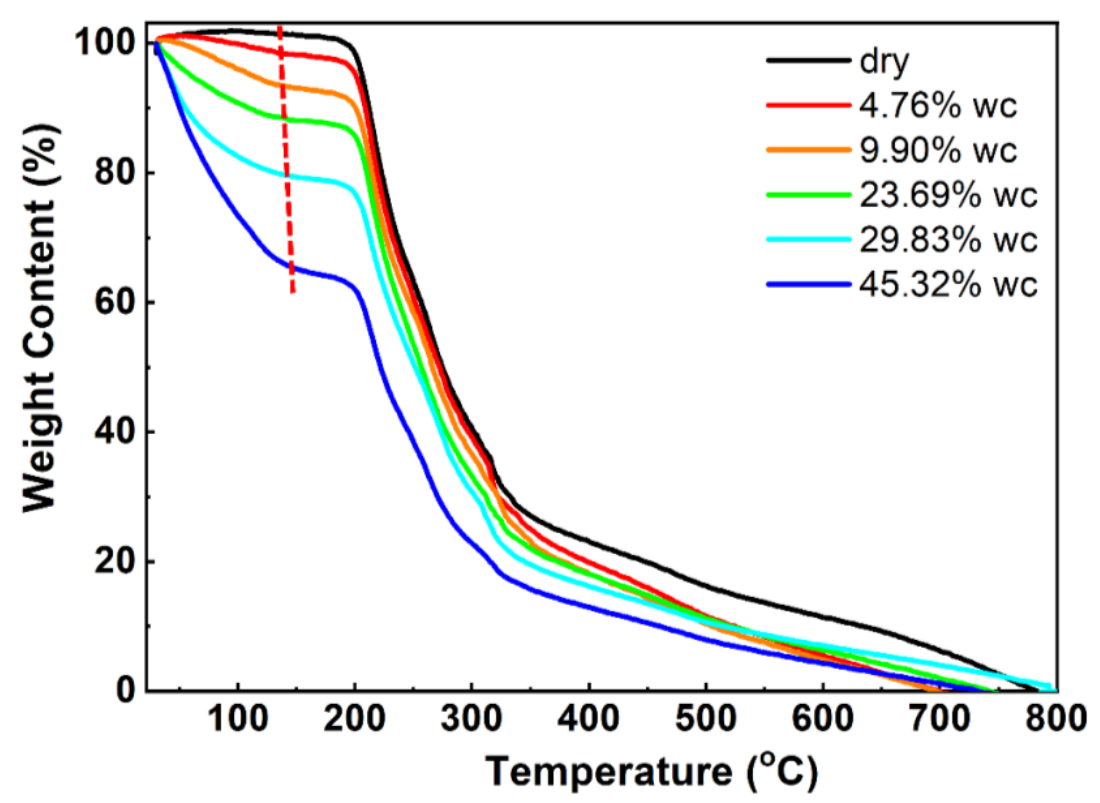

Fig. S11. TGA data of ADM-4CTAB MSCs with different water content.

The freezable water can be further distinguished from the non-freezable water using differential scanning calorimeter (DSC). According to the previous literatures ${ }^{3-5}$, the total water $\left(W_{t}\right)$ inside amphiphilic macromolecular polymer could clarified into three different states, which are named as nonfreezable water $\left(W_{n f}\right)$, freezable water $\left(W_{f}\right)$ (including freezable bound water and free water). The $W \mathrm{t}$ is the total water content calculated by equation $\left(W \mathrm{t}=\frac{m(\text { wet sample })-m(\text { dry sample })}{m(\text { wet sample })} \times 100 \%\right)$. $W_{f}$ could be calculated from equation $W_{f}=\frac{\Delta H_{f}}{\Delta H_{w}} \times 100 \%$. The $\Delta H_{w}$ is the standard melting enthalpy of 
pure bulk water equal to $333.5 \mathrm{~J} / \mathrm{mol}$. While the $\Delta H_{f}$ is the endothermic enthalpy of freezable water, which could be integrated from the melting endothermic peak on the DSC curve. The melting temperature of freezable bound water is usually lower than free bulk water, so these two endothermic peaks could be separated generally. However, here the melting endothermic peak of freezable bound water and free water were merged, only the trend could be observed after increased the total water content of bulk ADM-4CTAB MSCs that the merged melting endothermic peaks would shift to higher temperature near $0^{\circ} \mathrm{C}$ (Fig. S12), which indicated that the free water content increased. The merged peak here was assigned to total freezable water $W_{f}$. According to heating cycle in Fig. S12, the detailed water content of different water states and phase transition point were summarized into following Table S1.

Table S1. DSC data summary of MSCs with different water content calculated from heating cycle.

\begin{tabular}{|c|c|c|c|c|c|}
\hline Wt (\%) & $\begin{array}{l}\text { Freezable water melting } \\
\text { tempeture }\left({ }^{\circ} \mathrm{C}\right)\end{array}$ & $\begin{array}{l}\text { Freezable water melting } \\
\qquad \Delta H_{f}(\mathrm{~J} / \mathrm{g})\end{array}$ & $\begin{array}{r}W_{f} \\
(\%)\end{array}$ & $\begin{array}{l}W_{n f} \\
(\%)\end{array}$ & $\begin{array}{c}\text { Phase transition temperature } \\
\text { of MSCs }\left({ }^{\circ} \mathrm{C}\right)\end{array}$ \\
\hline 0.00 & N/A & 0 & 0 & 0 & 81.20 \\
\hline 4.74 & N/A & 0 & 0 & 4.74 & 50.54 \\
\hline 9.82 & -18.20 & 5.5763 & 1.67 & 8.14 & 32.19 \\
\hline 20.87 & -3.84 & 10.6631 & 3.20 & 17.68 & 19.38 \\
\hline 29.91 & 0.11 & 41.6044 & 12.47 & 17.43 & 16.62 \\
\hline 44.87 & 3.06 & 92.0948 & 27.61 & 17.25 & N/A* \\
\hline
\end{tabular}

(N/A: no available data can be obtained)
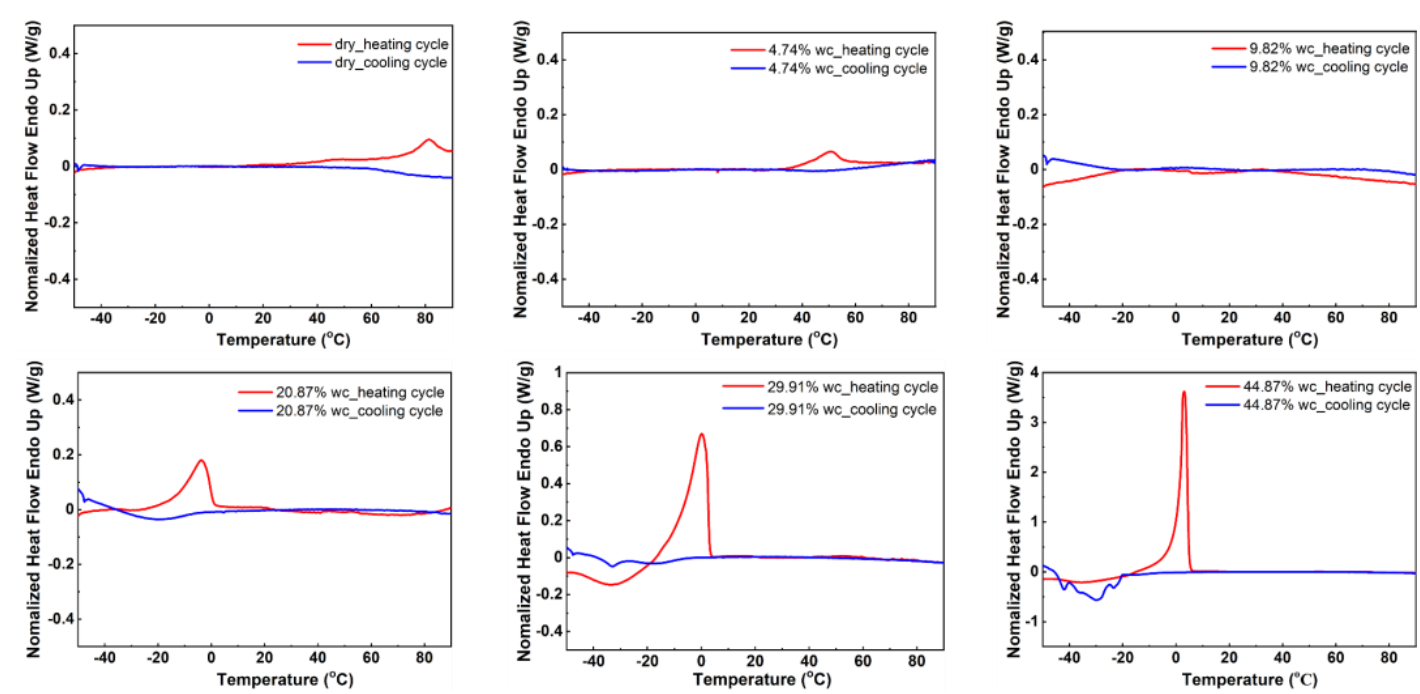

Fig. S12. DSC data of ADM-4CTAB MSCs with different water content. 


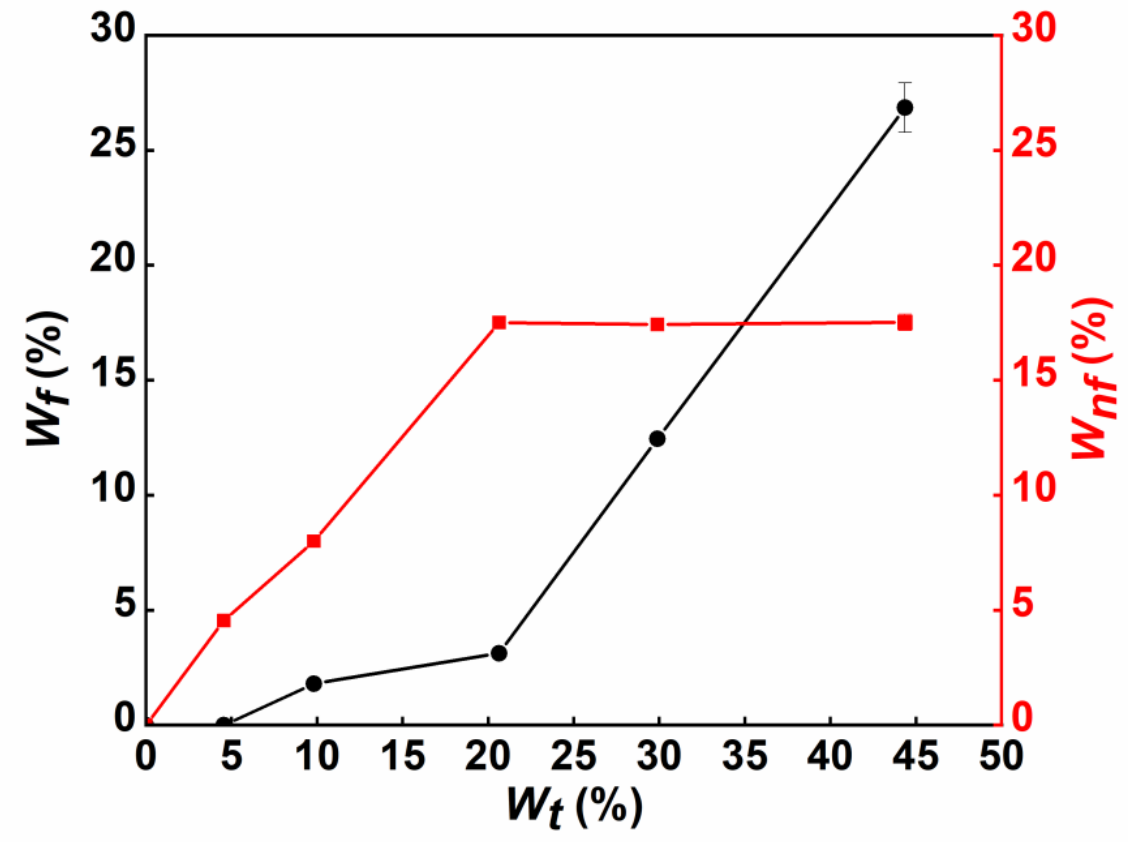

Fig. S13. The distribution changes of water states including non-freezable water $\left(W_{n f}\right)$ and freezable water $\left(W_{f}\right)$ inside ADM-4CTAB MSCs under different total water content $\left(W_{t}\right)$.

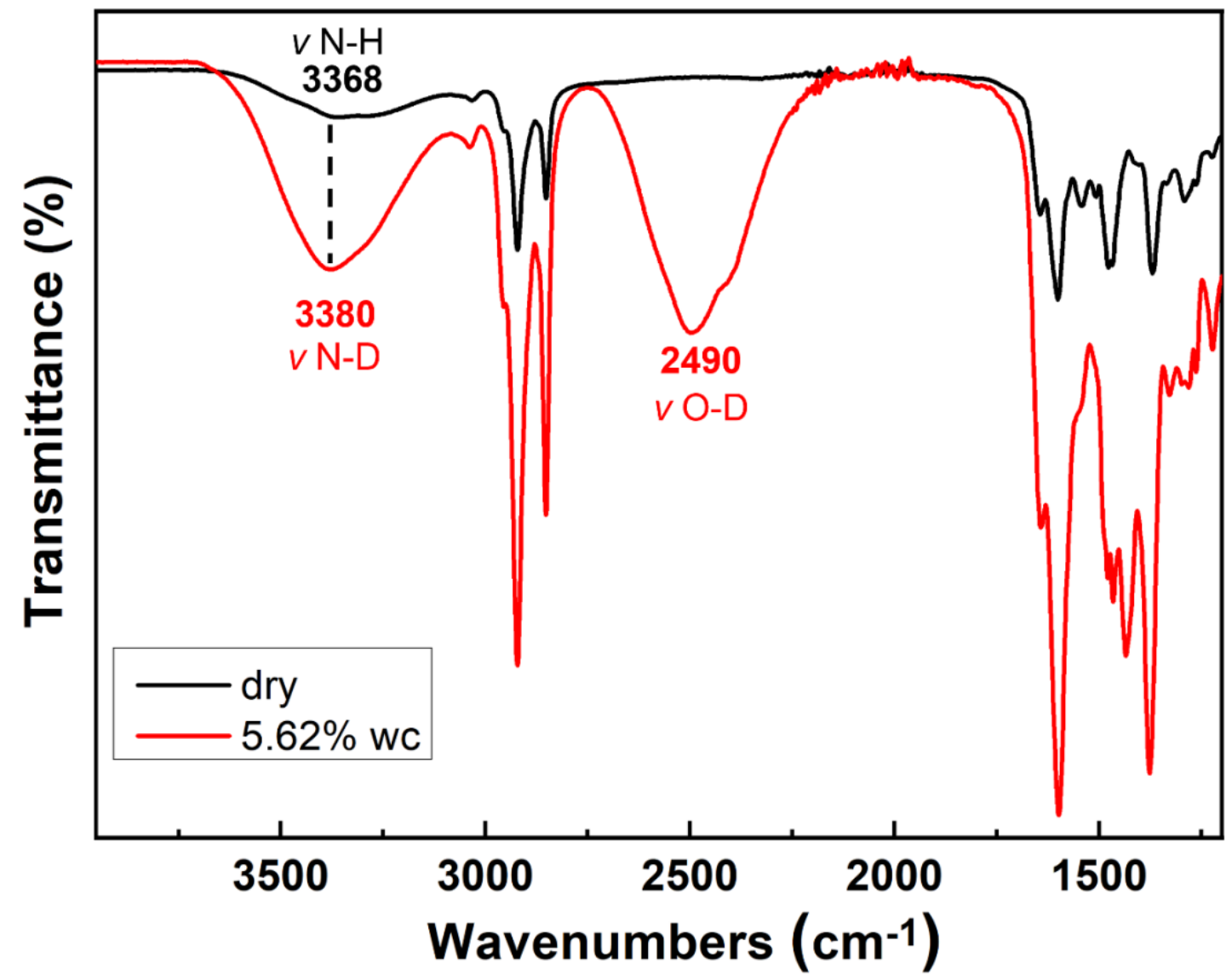

Fig. S14 ATR-FTIR spectrum of ADM-4CTAB MSCs with different water (D2O) content. 
5. Self-healing, adhesive and robust properties of materials processed from the MSCs
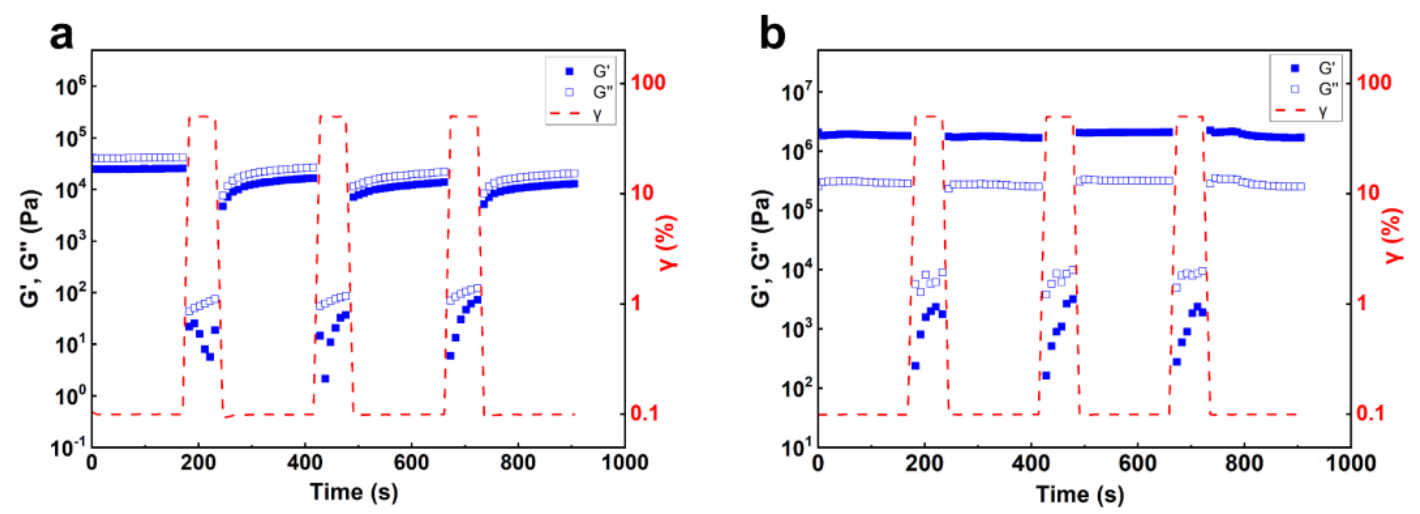

Fig. S15. The thixotropic behavior showing loss ( $\mathrm{G}^{\prime \prime}$, open data point) and storage moduli ( $\mathrm{G}^{\prime}$, filled data points) of bulk ADM-4CTAB MSCs with (a) $\sim 5 \%$ and (b) $5 \%$ water content.
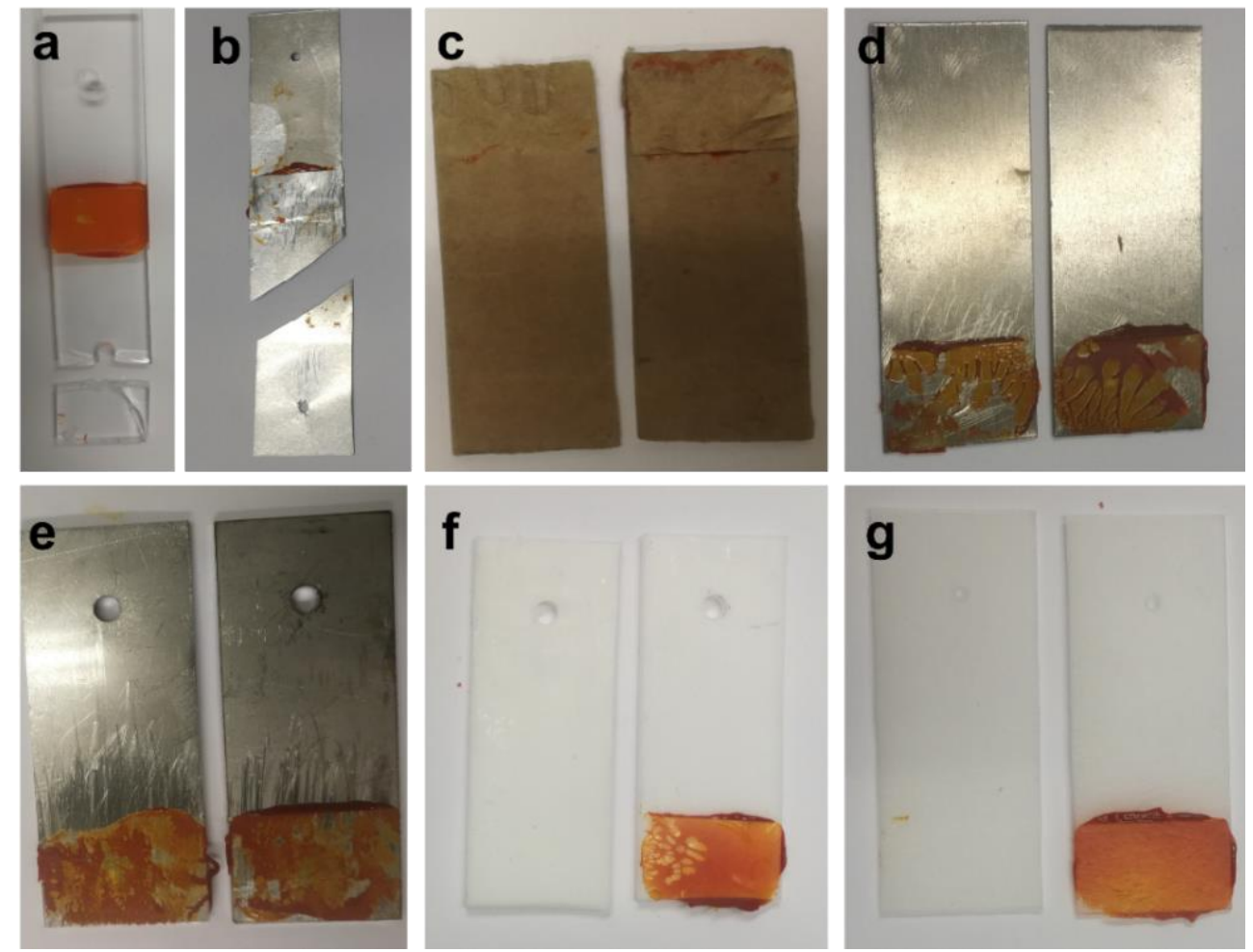

Fig. S16. Photos of (a) quartz glasses, (b) aluminum, (c) corrugated paper, (d) titanium, (e) steel, (f) PTFE, (g) PE specimens after lap-shear tensile stress tests. 

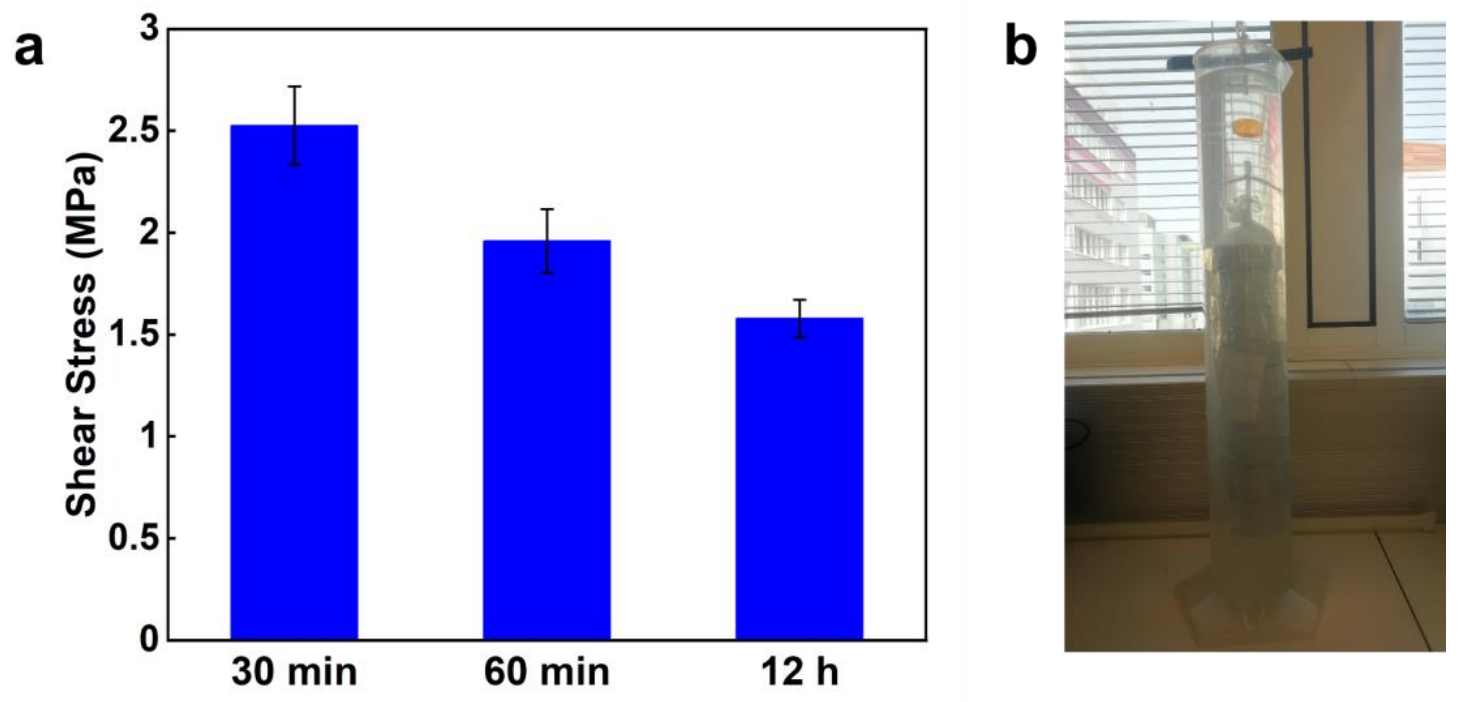

Fig. S17. (a) The lap-shear strength of ADM-4CTAB MSCs applied to steel substrates after immersed into water for different time. (b) ADM-4CTAB MSCs applied to quartz substrates could hold a $2 \mathrm{Kg}$ weight under water.

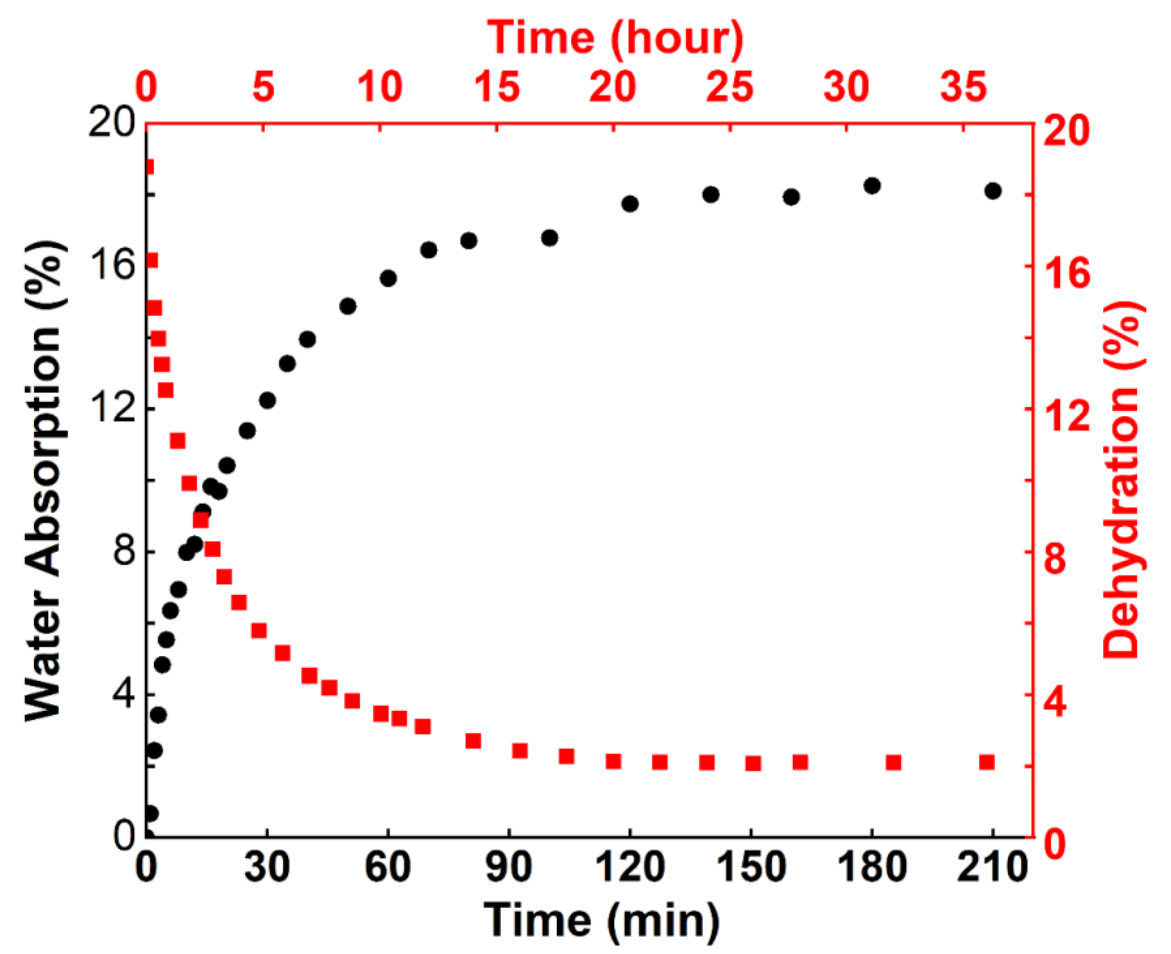

Fig. S18. The water absorption and dehydration curves of ADM-4DHDAB MSCs. 


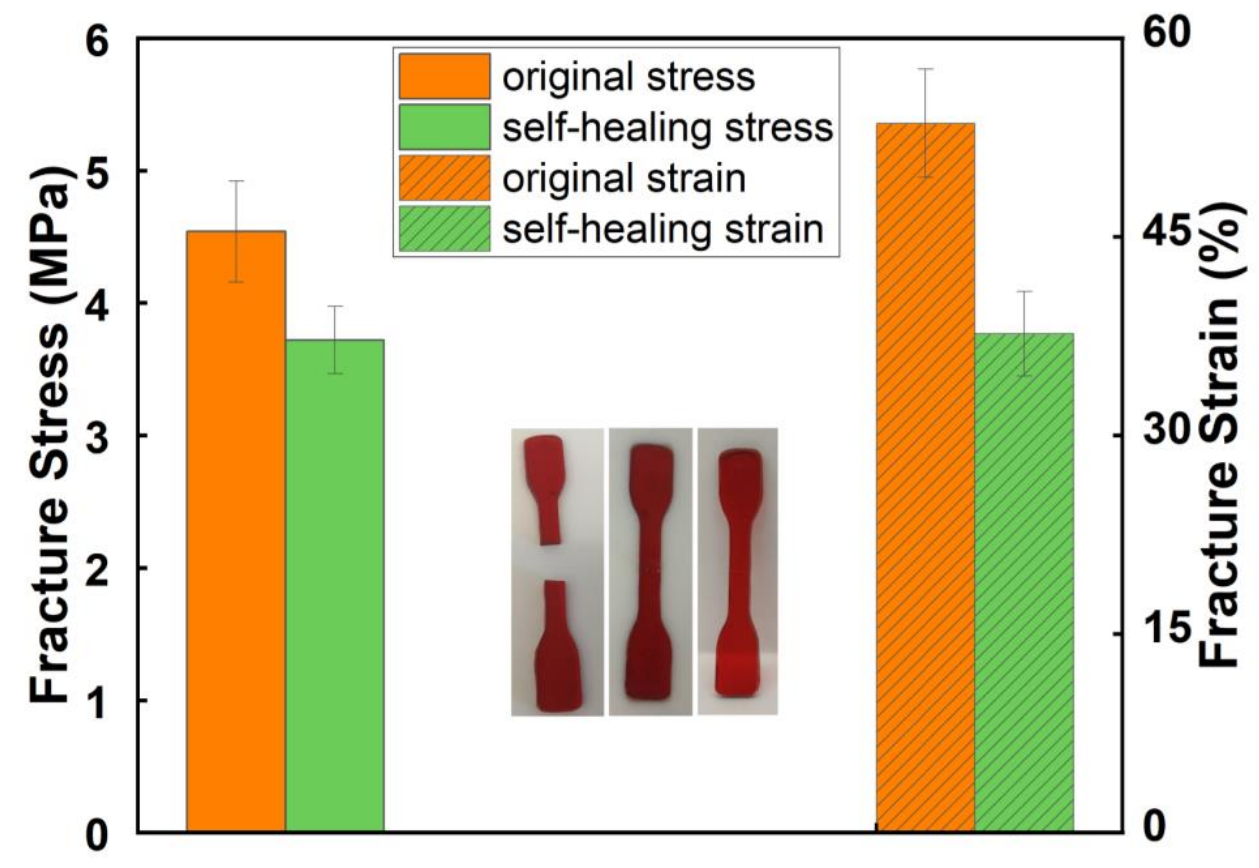

Fig. S19. Fracture stress and fracture strain changes of free-standing ADM-4CTAB MSCs between original and self-healing specimens. (RH $55 \%, 21^{\circ} \mathrm{C}$, insert photos: dumbbell-like free-standing MSCs specimens after cutting, self-healing with a drop of water and dried in air)

\section{Stability and responsiveness of the MSCs}

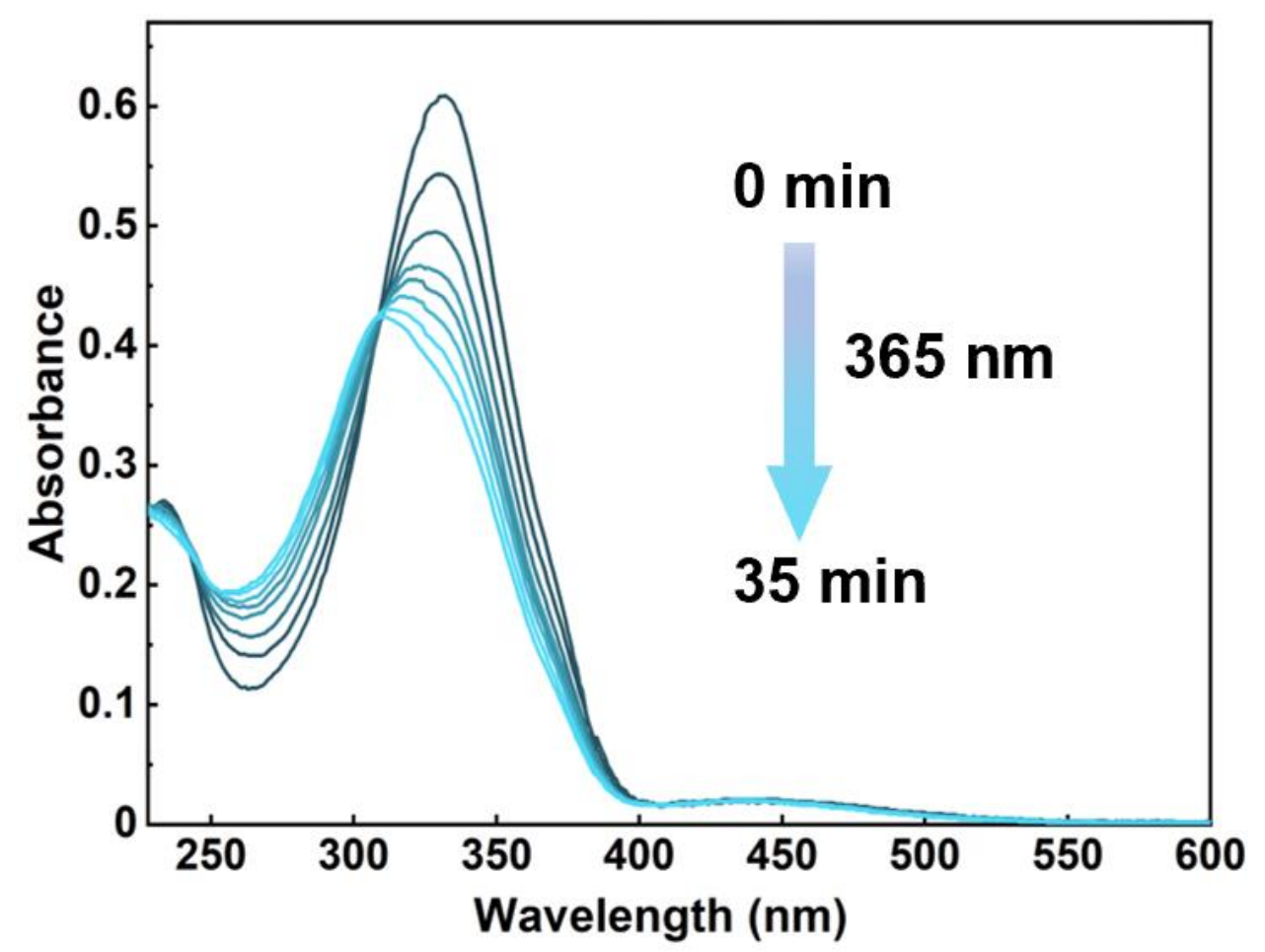

Fig. S20. UV-vis absorption spectrum of ADM aqueous solution under UV $365 \mathrm{~nm}$ irradiation $\left(2 \times 10^{-5} \mathrm{M}, \mathrm{H}_{2} \mathrm{O}, \mathrm{pH}=10\right)$. 

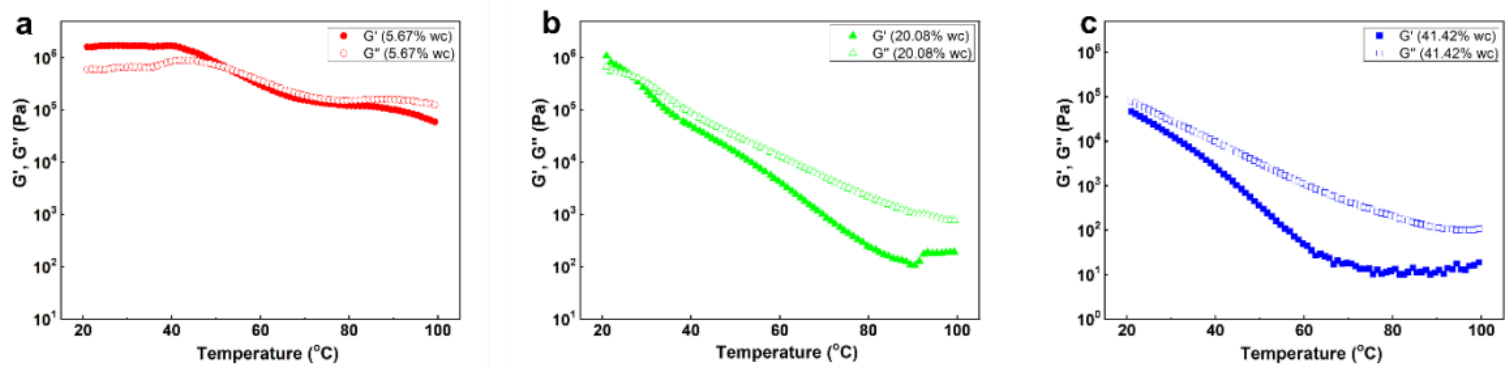

Fig. S21. Temperature-variable rheological properties showing loss ( $\mathrm{G}^{\prime \prime}$, open data point) and storage moduli ( $\mathrm{G}^{\prime}$, filled data points) of (a)-(c) bulk ADM-4CTAB MSCs with different water content (angular frequency $\omega=10 \mathrm{rad} / \mathrm{s}$, amplitude $\gamma=0.1 \%$ ).

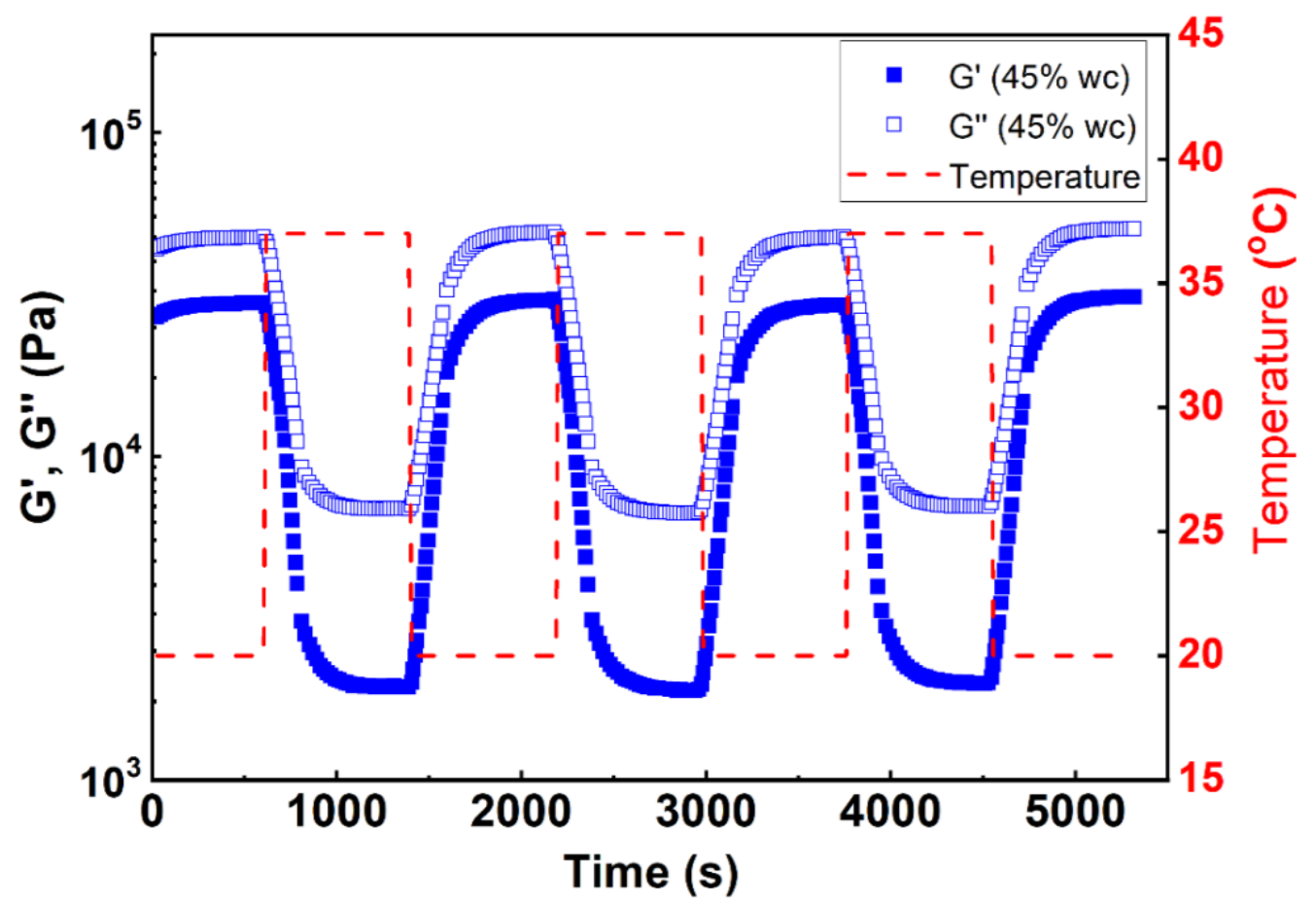

Fig. S22. Cyclic temperature-dependency sweep measurement of ADM-4CTAB MSCs with $\sim 45 \%$ water content. 


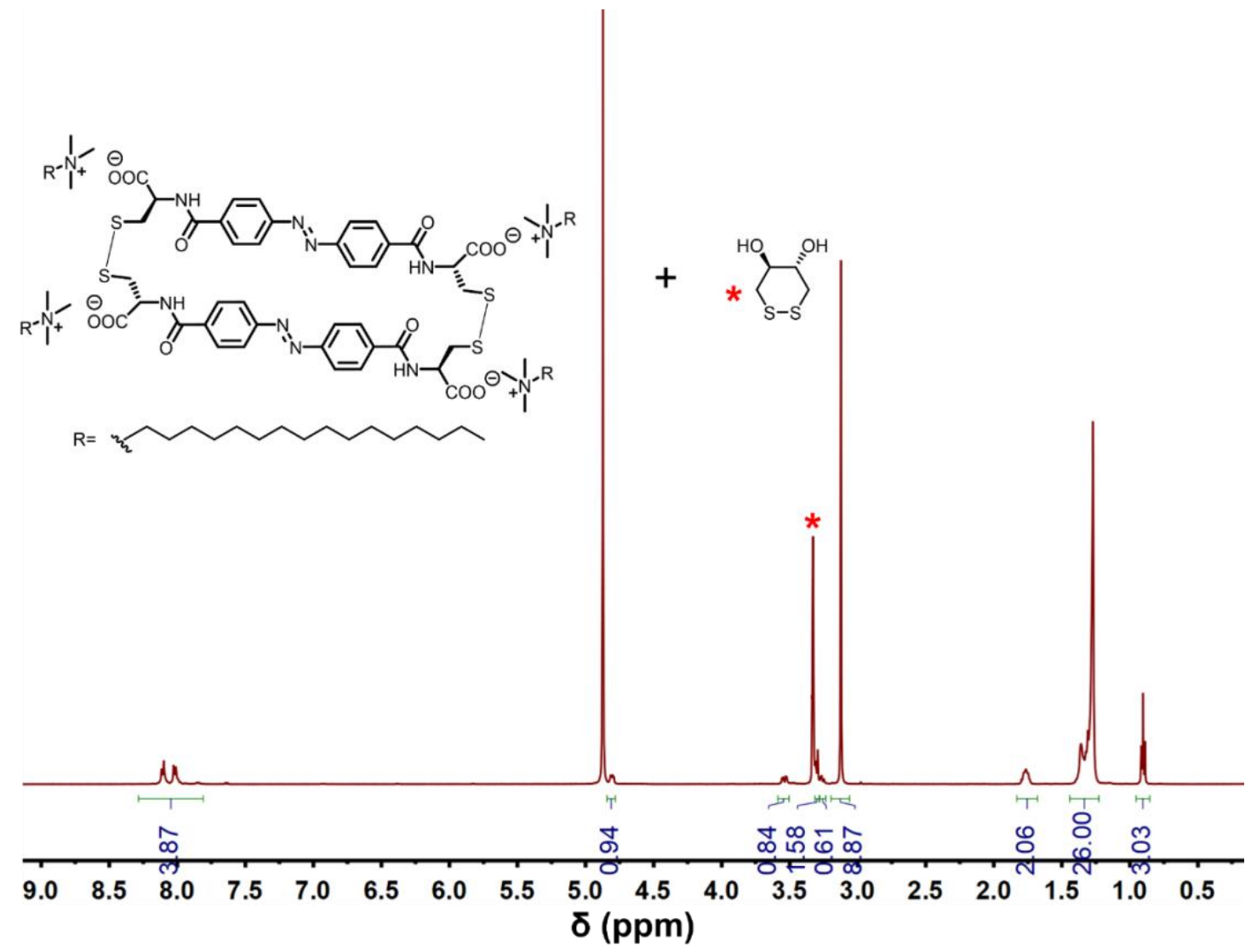

Fig. S23 ${ }^{1} \mathrm{H}$ NMR of ADM-4CTAB MSCs after reducing with DTT and oxidizing again with air (*: the oxidation product of DTT, $\mathrm{CD}_{3} \mathrm{OD}-\mathrm{d}^{4}, 500 \mathrm{MHz}, 298 \mathrm{~K}$ )

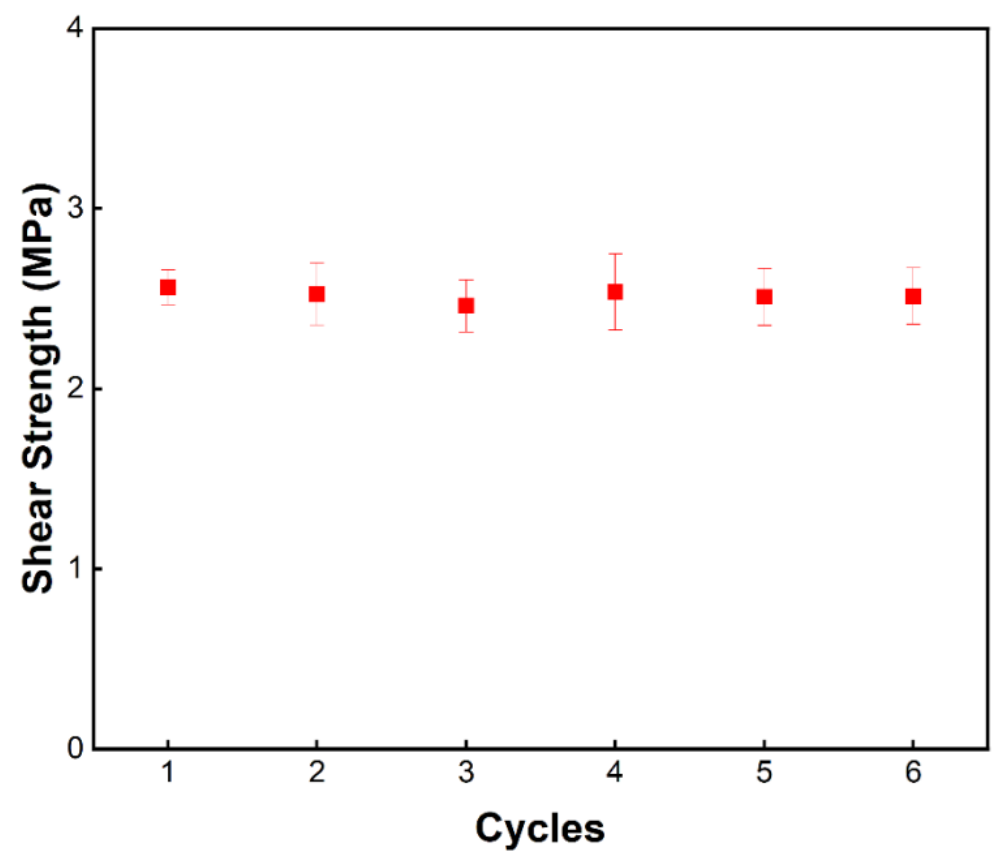

Fig. S24. The lap-shear strength of ADM-4CTAB MSCs apply for Titanium substrate for 6 cycles. 


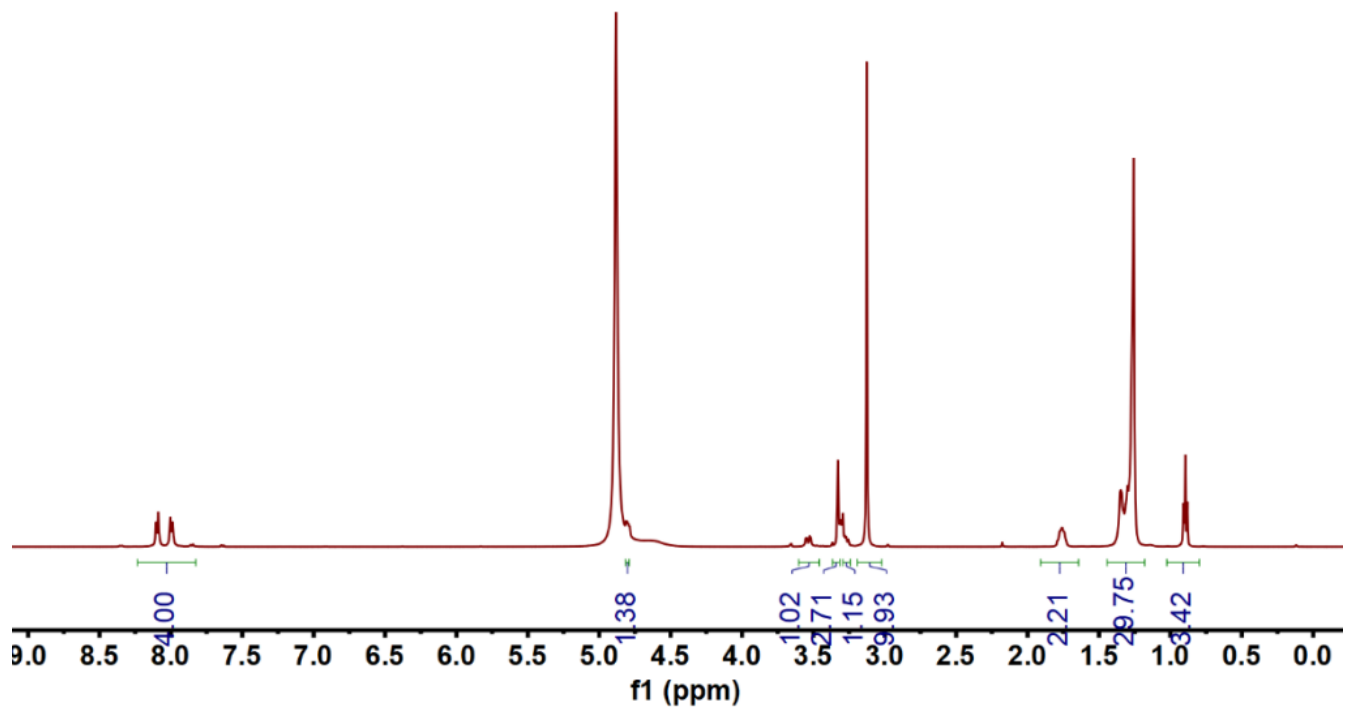

Fig. S25 ${ }^{1} \mathrm{H}$ NMR of lyophilic ADM-4CTAB MSCs after recycling for many times $\left(\mathrm{CD}_{3} \mathrm{OD}-\mathrm{d}^{4}\right.$, $500 \mathrm{MHz}, 298 \mathrm{~K}$ )

\section{Description of Supplementary Movies}

All the details for supplementary movies were provided in the following section, and some necessary information was also marked as related texts in the videos.

Movie S1. The movie shows the growth process of the coacervate droplets formed from ADM(1 $\mathbf{m M}) / \mathbf{C T A B} / \mathbf{1}: 4$ into water $(\mathrm{pH}=10)$ after mixing for $15 \mathrm{~min}, 30 \mathrm{~min}, 90 \mathrm{~min}$. The time-lapse video was recorded by a multimode microplate reader (Cyation 5, BioTek Instruments Inc.). The play speed of this video was sped up 50x.

Movie S2. The movie shows the underwater self-healing process of ADM-4CTAB MSCs. In order to easily visualize this process, half cylinder-like MSCs was colored by Brilliant blue R-250. Then the MSCs were cut into several pieces, the self-healing process were carried out by contacting fragments with tweezers in seconds. The play speed of this video was sped up 3x.

Movie S3. The movie shows two pieces of steel substrates jointed by ADM-4CTAB MSCs ( $45 \% \mathrm{wc}$, $30 \mathrm{mg}$, coating area: $10 \mathrm{~mm} \times 20 \mathrm{~mm}$ ), after dried in air overnight, which can hold a $16 \mathrm{Kg}$ load over one month. The play speed of this video was sped up $2 x$.

Movie S4. The movie shows tensile-stress test of ADM-4DHDAB MSCs with $\sim 2 \%$ water content. The test specimen was prepared in a dumbbell-like PTFE mould with standard JIS K6251-8 dimensions (length $16 \mathrm{~mm} \times$ width $4 \mathrm{~mm} \times$ thickness $2 \mathrm{~mm}$ ), the strain rates were set to $10 \mathrm{~mm} / \mathrm{min}$. The play speed of this video was sped up $8 x$. 


\section{References}

1. $\mathrm{Xu}, \mathrm{L}$. J. et al. A solvent-exchange strategy to regulate noncovalent interactions for strong and antiswelling hydrogels. Adv. Mater. 32, 2004579. (2020).

2. Kim, S. J., Lee, C. K. \& Kim, S. I. Characterization of the water state of hyaluronic acid and poly(vinyl alcohol) interpenetrating polymer networks. J. Appl. Polym. Sci. 92, 1467-1472. (2004).

3. Ping, Z. H., Nguyen, Q. T., Chen, S. M., Zhou, J. Q. \& Ding, Y. D. States of water in different hydrophilic polymers - dsc and ftir studies. Polymer 42, 8461-8467. (2001).

4. Muller-Plathe, F. Different states of water in hydrogels? Macromolecules 31, 6721-6723. (1998).

5. Takigami, S., Kimura, T. \& Nakamura, Y. The state of water in nylon-6 membranes grafted with hydrophilic monomers .2. Water in acrylic-acid, acrylamide and p-styrenesulphonic acid grafted nylon-6 membranes. Polymer 34, 604-609. (1993). 